\title{
Establishment of mouse expanded potential stem cells
}

\author{
Jian Yang ${ }^{\# 1}$, David J. Ryan\#1, Wei Wang ${ }^{\# 1}$, Jason Cheuk-Ho Tsang ${ }^{\# 1}$, Guocheng Lan\#2, \\ Hideki Masaki ${ }^{\# 3}$, Xuefei Gao' ${ }^{1}$, Liliana Antunes ${ }^{1}$, Yong Yu ${ }^{1}$, Zhexin Zhu' ${ }^{1}$, Juexuan Wang ${ }^{1}$, \\ Aleksandra A. Kolodziejczyk ${ }^{1,4}$, Lia S. Campos ${ }^{1}$, Cui Wang ${ }^{1}$, Fengtang Yang ${ }^{1}$, Zhen \\ Zhong $^{5}$, Beiyuan Fu' ${ }^{1}$, Melanie A. Eckersley-Maslin ${ }^{6}$, Michael Woods ${ }^{1}$, Yosuke Tanaka ${ }^{7,8,9}$, \\ Xi Chen ${ }^{1}$, Adam C. Wilkinson ${ }^{7,8}$, James Bussell ${ }^{1}$, Jacqui White ${ }^{1}$, Ramiro Ramirez-Solis ${ }^{1}$, \\ Wolf Reik $^{1,6}$, Berthold Göttgens ${ }^{7,8}$, Sarah A. Teichmann ${ }^{1,4}$, Patrick P. L. Tam ${ }^{10,11}$, Hiromitsu \\ Nakauchi ${ }^{3,12}$, Xiangang Zou ${ }^{2}$, Liming $\mathrm{Lu}^{1,13}$, and Pentao Liu ${ }^{1}$ \\ ${ }^{1}$ Wellcome Trust Sanger Institute, Hinxton, Cambridge CB10 1HH, UK \\ ${ }^{2}$ Cancer Research UK Cambridge Institute, University of Cambridge, Li Ka Shing Centre, \\ Cambridge CB2 ORE, UK \\ ${ }^{3}$ Division of Stem Cell Therapy, Institute of Medical Science, University of Tokyo, Tokyo 108-8639, \\ Japan \\ ${ }^{4}$ European Molecular Biology Laboratory, European Bioinformatics Institute, Hinxton, Cambridge \\ CB10 1SD, UK \\ ${ }^{5}$ Medical Research Council Laboratory of Molecular Biology, Francis Crick Avenue, Cambridge \\ CB2 OQH, UK \\ ${ }^{6}$ Epigenetics Programme, The Babraham Institute, Cambridge CB22 3AT, UK \\ ${ }^{7}$ Cambridge Institute for Medical Research, University of Cambridge, Cambridge CB2 OXY, UK
}

Corresponding author: Correspondence to Pentao Liu.

Data availability

The sequencing data (RNA-seq, ChIP-seq) have been deposited in the European Nucleotide Archive under accession number ERP005641. All other data are available from the corresponding author upon reasonable request.

Accessions

Primary accessions

European Nucleotide Archive

\section{ERP005641}

Contributions

D.J.R. and W.W. developed EPSCM and derived mouse EPSC lines. J.Y. performed most of the experiments, made the final figures and edited the manuscript. J.C.-H.T performed and analysed the RNA-seq and chromatin immunoprecipitation followed by sequencing (ChIP-seq) experiments, produced the genomics figures and wrote the manuscript. G.L. performed most of the injections. H.M. performed independent EPSC injection experiments at Nakauchi laboratory. X.G., L.A., Y.Y., Z. Zhu, J.W., A.A.K. and C.W. performed genotyping, additional line derivation, FACS, sequencing and chimaera experiments. L.S.C. interpreted the histology data. F.Y. and B.F. karyotyped EPSC and ES cell lines. Z.Zho. performed confocal imaging and interpretation. M.A.E.-M. and W.R. provided DNA methylation data. M.W., Y.T. and A.C.W. performed additional experiments. X.C. analysed TSC RNA-seq data. J.B., J.W., R.R.-S., W.R., B.G., S.A.T., H.N. and X.Z. provided microinjection resources, sequencing and other support. L.L. and P.P.L.T. contributed intellectually and assisted with the revision of the manuscript. P.L. devised the concept, supervised the overall research project and prepared the manuscript for publication.

Competing interests

The Genome Research Limited has filed a provisional patent application that covers the derivation and maintenance of expanded potential stem cells (European patent application number 15797300.9-1402). P.L., D.J.R., J.Y., W.W. and X.G. are listed as inventors. 
${ }^{8}$ Wellcome Trust-MRC Cambridge Stem Cell Institute, University of Cambridge, Cambridge CB2 OXY, UK

${ }^{9}$ Division of Cellular Therapy, Institute of Medical Science, University of Tokyo, Tokyo 108-8639, Japan

${ }^{10}$ Embryology Unit, Children's Medical Research Institute, University of Sydney, Westmead, New South Wales 2145, Australia

${ }^{11}$ School of Medical Sciences, Sydney Medical School, University of Sydney, Westmead, New South Wales 2145, Australia

${ }^{12}$ Institute for Stem Cell Biology and Regenerative Medicine, Department of Genetics, Stanford University School of Medicine, Stanford, California 94305-5461, USA

${ }^{13}$ Shanghai Institute of Immunology, Shanghai, Jiaotong University School of Medicine, Shanghai 200025, China

\# These authors contributed equally to this work.

\section{Abstract}

Mouse embryonic stem cells derived from the epiblast 1 contribute to the somatic lineages and the germline but are excluded from the extra-embryonic tissues that are derived from the trophectoderm and the primitive endoderm 2 upon reintroduction to the blastocyst. Here we report that cultures of expanded potential stem cells can be established from individual eight-cell blastomeres, and by direct conversion of mouse embryonic stem cells and induced pluripotent stem cells. Remarkably, a single expanded potential stem cell can contribute both to the embryo proper and to the trophectoderm lineages in a chimaera assay. Bona fide trophoblast stem cell lines and extra-embryonic endoderm stem cells can be directly derived from expanded potential stem cells in vitro. Molecular analyses of the epigenome and single-cell transcriptome reveal enrichment for blastomere-specific signature and a dynamic DNA methylome in expanded potential stem cells. The generation of mouse expanded potential stem cells highlights the feasibility of establishing expanded potential stem cells for other mammalian species.

We sought to develop an in vitro culture condition that enables the establishment of pluripotent stem cells from cleavage stage mouse embryos. We posited that such pluripotent stem cells might possess expanded potential to descendants of both the trophectoderm and the inner cell mass, similar to four-cell or eight-cell embryo blastomeres. To halt blastomere differentiation and to enable cell line derivation, we hypothesized that modulation of signalling pathways affecting trophectoderm/inner cell mass segregation might be the key. Previous studies have established the roles of mitogen-activated protein kinases (MAPKs), Src, Hippo pathways and poly-ADP-ribosylation (PARP) regulators in this process3-7. In particular, Src blockade partly arrested morula and affected trophectoderm and primitive endoderm (PrE) in the blastocyst5. Genetic inactivation of Parp1/2 and tankyrase 1/2 (Tnks/ Tnks2) resulted in trophoblastic differentiation in embryonic stem (ES) cells and placenta defects, respectively, whereas deletion of Parg, which encodes poly(ADP-ribose) glycohydrolase that catalyses the hydrolysis of poly(ADP-ribose), led to loss of the trophectoderm and of the ability to generate trophoblast stem cells (TSCs)6,7. Wnt 
signalling is implicated in the earliest development of vertebrates8 and of trophoblasts.

Importantly, signalling cross-talks are prevalent between Wnt and MAPK pathways via Yap1, the key effector of Hippo pathway9-11.

We therefore elected to block blastomere differentiation by targeting MAPKs, Src and Wnt/ Hippo/TNKS1/2. For MAPKs, we used Mek1 inhibitor PD0325901, JNK Inhibitor VIII and p38 inhibitor SB203580. For Src kinase, we selected A-419259, a potent pyrrolopyrimidine inhibitor12. XAV939 was chosen to inhibit Parp family members TNKS1/2 and to stabilize AXIN, the concentration-limiting component of $\beta$-catenin and Yap destruction complex11,13. XAV939 also suppresses Yap1 activities via angiomotin14, and thus potentially modulates multiple targets relevant to trophectoderm/inner cell mass segregation. GSK3 inhibitor CHIR99021 was included, as it is an ES cell 2i medium component and improves cell culture robustness whereas its pro-pluripotency effect appears to be partly redundant with the presence of PD0325901 or leukaemia inhibitory factor (LIF)15,16. The combination of CHIR99021 and XAV939 has been used to culture pluripotent stem cells17,18. Finally, we chose LIF as it may promote the rare totipotent cells in mouse ES cell culture 19. Hereafter, we refer to the medium containing this cocktail of inhibitors and LIF as expanded potential stem cell medium (EPSCM), and used it for derivation of EPSC lines and their maintenance.

We first derived cell lines directly from eight-cell blastomeres in EPSCM (Fig. 1a). From 32 blastomeres, we obtained nine outgrowths consisting of Oct $4^{+} \mathrm{Cdx} 2^{-}$cells, and established eight stable EPSC lines. No outgrowths were obtained in M15 with $15 \%$ serum $(n=32)$ or 2i/LIF $(n=32)$. Stable EPSC lines could also be established de novo from whole four-cell or eight-cell embryos (Extended Data Fig. 1a and Supplementary Video) with an efficiency of $20 \%$ in feeder-free cultures, and up to $100 \%$ on SNL feeder cells. The established stem cell lines expressed pluripotency genes similar to conventional ES cells, had a normal karyotype, formed teratomas with multiple tissue types and contributed to the somatic and germline lineage in chimaeras (Extended Data Fig. 1b-g). Remarkably, once injected into morulas, EPSCs $\left(\mathrm{mCherry}^{+}\right)$contributed both to the inner cell mass and to the trophectoderm (Fig. $1 b)$.

After five passages in EPSCM, conventional ES cells (AB2.2 and E14Tg2a) or iPS cells also acquired an expanded potential to contribute to the $\mathrm{Cdx} 2^{+}$cells in the trophectoderm (Fig. 1b, c and Extended Data Fig. 1h). Notably, when EPSCs were returned to 2i/LIF medium, they lost the expanded potential and reverted to ES cells (Fig. 1b). The ES cell-EPSCs, similar to de novo EPSCs, shared many pluripotency properties, including robust Oct4-GFP (also known as Pou5f1-GFP) and Rex1-GFP(also known as Zfp42-GFP) expression, comparable pluripotency and lineage-specific gene expression, Oct4 distal enhancer predilection, tissue contribution in chimaeras, biallelic $\mathrm{X}$-linked gene activation in female EPSCs (Extended Data Figs 1i-k and 2a-d) and efficient Rosa26 locus gene targeting (around 50\%). Biochemically, the inhibitors in EPSCM effectively targeted their intended kinases (Extended Data Fig. 2e). Importantly, XAV939 increased axin (Extended Data Fig. 2e), which led to lower active $\beta$-catenin in the nucleus (Extended Data Fig. 2f) and no Wnt activity detectable by TOPflash assay (Extended Data Fig. 2g), despite the presence of CHIR99021 in EPSCM. EPSCs were responsive to LIF-induced JAK/STAT signalling 
(Extended Data Fig. 2h, i) but were unresponsive to FGFR or ALK5 inhibition, similar to 2i/LIF ES cells (Extended Data Fig. 2j).

In post-implantation embryos, donor mCherry ${ }^{+}$EPSCs were found both in the embryo proper and in the trophectoderm-derived Elf5 ${ }^{+}$extra-embryonic ectoderm (ExE) region20 in about $35 \%$ of the 6.5 days post-coitum (d.p.c.) chimaeras (78 out of 225) (Fig. 2a and Extended Data Fig. 3a). By contrast, 2i/LIF ES cells did not contribute to the ExE. Other chimaeras had EPSC contributions either in the embryo proper $(n=143)$ or, in rare cases $(n$ $=4$ ), predominantly in the ExE (Extended Data Fig. 3a), indicating overall less efficient differentiation of EPSCs to trophectoderm derivatives.

In 14.5 d.p.c. chimaeras, EPSC descendants were found in the embryo proper and in the extra-embryonic tissues, the latter confirmed by flow cytometry analysis of placenta cells (Fig. 2b and Extended Data Fig. 3b, c). The mCherry ${ }^{+}$(EPSC-derived) and mCherry ${ }^{-}$(host) placenta cells expressed similar trophoblast genes (Fig. 2c). Many EPSC-derived mCherry ${ }^{+}$ placenta cells isolated from chimaeras, but not those derived from $2 \mathrm{i} / \mathrm{LIF}$ ES cells, were polyploid (Extended Data Fig. 3d), similar to the host trophoblasts that are formed by endoreplication and cell fusion. The EPSC-derived cells expressed trophoblast markers Tfap2c, Gcm1, Ezrin and Cytokeratin 7 (CK7) (Fig. 2d and Extended Data Figs 3e and 4a). Finally, we excluded the possibility of cell fusion between the EPSC-derived cells and host trophoblasts by showing the absence of cells with both the host and EPSC genotypes (EPSCs were tagged by $S B 10$ at the Rosa26 locus) (Extended Data Fig. 4b).

In the yolk sac of 14.5 d.p.c. chimaeras, ES cells only contributed to extra-embryonic mesoderm, whereas descendants of EPSCs were found in both the extra-embryonic endoderm (derived from PrE) and the mesoderm cell layers (endothelial cells and mesothelial cells) (Extended Data Fig. 4c). The expanded potential of EPSCs was further demonstrated by the contribution of a single EPSC in chimaeras generated in eight-cell embryo hosts (Extended Data Fig. 5). Finally, we showed that as few as three inhibitors in EPSCM could confer substantial levels of expanded potential to ES cells both in vitro and in vivo (Extended Data Fig. 6).

We next analysed the molecular characteristics of EPSCs by RNA sequencing (RNA-seq). In hierarchical clustering, the transcriptomes of EPSCs and ES cells were segregated by the maintenance conditions, independently of derivation methods or culture history (Fig. 3a). Transcriptomes of EPSCs and ES cells (G4) maintained in 2i/LIF and M15 (ref. 21) were further compared by single-cell RNA-seq (scRNA-seq) (Extended Data Fig. 7a). In principal component analysis (PCA), individual cells were segregated by their culture conditions (Extended Data Fig. 7b and Supplementary Table 1). Gene Ontology term enrichment analysis revealed that $2 \mathrm{i} / \mathrm{LIF}$ ES cells were enriched for genes of oxidative metabolic processes22 (Extended Data Fig. 7c and Supplementary Table 1), whereas EPSCs were preferentially featured with genes of transcriptional regulation and embryonic development, particularly placental development.

Expression variability of key pluripotency genes revealed that EPSCs had similar transcriptomic homogeneity to 2i/LIF ES cells, and importantly, EPSCs did not appear to 
harbour lineage-predisposed subpopulations (Fig. 3b and Extended Data Fig. 7d). We next compared EPSCs with mouse pre-implantation embryos23, which showed EPSCs were separated from the native blastomeres in PCA (Fig. 3c). Yet, in the major embryonic development component (PC1), EPSCs were in the range of four-cell blastomeres (Extended Data Fig. 7e). Blastomeric stage-specific gene set enrichment analysis also revealed more enrichment of early pre-implantation (zygote, two-cell and four-cell) signatures in EPSCs than 2i/LIF ES cells (Fig. 3d). A recent epigenomic study in blastomeres identified that Nr5a2 (also known as Lrh1) and Rarg, both highly expressed in two-cell to eight-cell blastomeres23,24, are key determinants for trophectoderm/inner cell mass segregation 24 . Interestingly, EPSCs highly expressed both $\mathrm{Nr} 5 \mathrm{a} 2$ and Rarg whereas 2i/LIF ES cells only expressed $\mathrm{Nr}_{\mathrm{r}} \mathrm{a} 2$ (Extended Data Fig. 7d). Similarly, EPSCs, but not 2i/LIF ES cells, had high levels of Aire, Thap11 (also known as Ronin) and Lin28 (also known as Lin28a) (Extended Data Fig. 7d and Supplementary Table 1), which are also highly expressed in blastomeres. Finally, EPSCs were transcriptionally distinct from the recently reported rare totipotent-like ES cell subpopulations (MERV-TdTomato ${ }^{+}$and Hhex-Venus ${ }^{+}$)19,25 or in vivo reprogrammed iPS cells (iviPSCs)26 (Extended Data Fig. 8a, b).

EPSCs, in contrast to 2i/LIF ES cells27, displayed high expression of genes of DNA methyltransferases (Dnmt1, Dnmt3a and Dnmt3b) and demethylating enzymes (Tet1, Tet2 and $T d g$ ) (Extended Data Fig. 7d), similar to blastomeres of four-cell to eight-cell embryos23. EPSCs showed an intermediate level of 5-methylcytosine (5mC) compared with 2i/LIF and M15 ES cells (Fig. 3e), but had strikingly higher levels of 5-

hydroxymethylcytosine (5hmC) (Fig. 3f), similar to the active DNA demethylation in blastomeres.

EPSCs had more genes $(6,224$ versus 3,968) associated with both H3K4me3 and H3K27me3 (bivalent) than 2i/LIF ES cells22 (Fig. 3g, Extended Data Fig. 8c, d and Supplementary Table 2), but H3K4me3 signals at key pluripotency loci such as Oct4, Sox 2 and Nanog were similar to those in 2i/LIF ES cells (Extended Data Fig. 8e). EPSC-specific bivalent genes (Supplementary Table 2) were enriched in developmental processes (Extended Data Fig. 8f, g). The H3K4me3 patterns of certain loci in EPSCs resembled that of eight-cell embryos28 (Extended Data Fig. 8g). Furthermore, a unique super-enhancer at the Gata3 locus, which is important in the trophectoderm, was identified in EPSCs (Fig. 3h and Extended Data Fig. 8h). Despite the similarities, the in vitro cultured self-renewing EPSCs were different from the in vivo blastomeres.

Three stem cell lines, ES cells, TSCs and extra-embryonic endoderm stem (XEN) cells, could be derived from the blastocyst. In TX medium29, EPSCs, but not ES cells, produced TSC-like Cdx2 ${ }^{+}$cells (Extended Data Fig. 9a). We established TSC-like cell lines from Cdx2-GFP reporter EPSCs in TX medium after fluorescence-activated cell sorting (FACS) purification (Fig. 4a). These cells proliferated well, and expressed high levels of TSC genes but not Oct 4 or markers of other lineages, and showed DNA demethylation at the Elf5 locus (Fig. 4b, Extended Data Fig. 9b-d and Supplementary Table 3). These cells, named EPSC TSCs, differentiated into trophoblasts in vitro and formed trophoblastic giant cell-rich haemorrhagic lesions in vivo (Fig. $4 \mathrm{c}$ and Extended Data Fig. 9e-i). Similar to TSCs, Cdx2 ${ }^{+}$ and $\mathrm{Elf5}^{+}$descendants from EPSC TSCs were found in the trophectoderm and in the ExE 
region in 6.5 d.p.c. chimaeras (Fig. 4d, e), confirming derivation of bona fide TSCs from EPSCs.

EPSCs, but not ES cells, contributed to the yolk sac endoderm (Extended Data Figs $4 \mathrm{c}$ and 6f), which is derived from the PrE (or hypoblast) in the blastocyst. We generated eight XEN cell lines from Gata6H2B-Venus reporter EPSCs30. These EPSC-XEN cells expressed PrEassociated genes (Extended Data Fig. 10a-c) and contributed to putative PrE derivatives in chimaeras (Extended Data Fig. 10d).

Finally, we genetically confirmed that overexpressing axin1 in 2i/LIF ES cells was sufficient for them to acquire the expanded potential and to contribute to the ExE in chimaeras (Extended Data Fig. 10e, f).

By modulating key developmental pathways, we obtained self-renewing stem cell lines with the expanded potential for all blastocyst cell lineages derived from mouse early preimplantation embryos, from ES cells and iPS cells. These insights may expedite the generation of similar EPSCs from other mammalian species for which ES cells or iPS cells are not yet available.

\section{Methods}

\section{Ethics statement}

All experimental procedures were performed in accordance with UK Home Office regulations and the Animals (Scientific Procedures) Act 1986 (licence number 70/8387 and 80/2552). All experimental protocols were approved by the Animal Welfare and Ethical Review Body of the Wellcome Genome Campus and the University of Cambridge CRUK Cambridge Institute. At the end of the study, mice were euthanized by cervical dislocation, in accordance with stated UK Home Office regulations.

Mice

All C57BL/6J, CD1 and C57BL6/JCBA F1 mice were from Charles River Laboratories.

\section{Culturing mouse EPSCs, ES cells and iPS cells}

Mouse ES cells were cultured in standard N2B27-2i/LIF or in M15 media-KnockOut DMEM (Invitrogen), 15\% fetal bovine serum (FBS, Hyclone), $1 \times$ glutamine-penicillinstreptomycin (GPS, Invitrogen), $1 \times$ non-essential amino acids (Invitrogen), $0.1 \mathrm{mM} \beta$ mercaptoethanol ( $\beta$-ME, Sigma) and $10^{3} \mathrm{U} \mathrm{ml}^{-1} \mathrm{hLIF}$ (Millipore, catalogue number LIF1010, lot 2199745). Mouse EPSCs were cultured in EPSCM: DMEM/F12 (Invitrogen), $20 \%$ KnockOut Serum Replacement (KSR, Invitrogen), $1 \times$ GPS, $1 \times$ non-essential amino acids, $0.1 \mathrm{mM} \beta$-ME and $10^{3} \mathrm{U} \mathrm{ml}^{-1} \mathrm{hLIF}$ supplemented with the following small-molecule inhibitors: CHIR99021 (Tocris, $3 \mu \mathrm{M}$, catalogue number 4423, lot 3A/167378), PD0325901 (Tocris, $1 \mu \mathrm{M}$, catalogue number 4192, lot 2), JNK Inhibitor VIII (Tocris, $4 \mu \mathrm{M}$, catalogue number 3222, lot 4A/166244), SB203580 (Tocris, $10 \mu \mathrm{M}$, catalogue number 1402, 4A/ 167379), A-419259 (Santa Cruz, $0.3 \mu \mathrm{M}$, catalogue number sc-361094, lot D2214) and XAV939 (Sigma, $5 \mu$ M, catalogue number X3004, lot 094M4730V). Apart from the abovementioned inhibitors, alternative inhibitors were also tested in EPSCM, including Src 
inhibitor CGP77675 (Sigma, $1 \mu$ M, catalogue number SML0314) and Wnt inhibitor IWR-1 (Sigma, $2.5 \mu \mathrm{M}$, catalogue number 10161). Other inhibitors used were JAK inhibitor I (Santa Cruz, $0.6 \mu \mathrm{M}$, catalogue number sc-204021), FGFR inhibitor SU5402 (Tocris, $2 \mu \mathrm{M}$, catalogue number 3300), TGF- $\beta$ receptor inhibitors A83-01 (Tocris, $0.5 \mu \mathrm{M}$, catalogue number 2939) and SB505124 (ALK5 inhibitor, Tocris, $1 \mu \mathrm{M}$, catalogue number 3263).

To convert mouse ES cells to EPSCs, ES cells were plated on mitomycin C-inactivated SNL feeder cells in EPSCM. Cells were passaged every 3 days, usually after five passages, which took about 15 days; the cells functionally acquired expanded potential, although no obvious cell growth difference from ES cells in serum LIF or 2i/LIF was observed. Mouse EPSCs were routinely cultured on inactivated SNL feeder cells and were passaged every 3-4 days at a ratio of 1:3 by a brief PBS wash followed by single-cell dissociation using Accutase (Merck Millipore, SCR005). For chimaeras, EPSCs or ES cells were usually made to express a CAG-mCherry, EF-1a-H2B-mCherry and CAG-H2B-mCherry expression cassette by piggyBac transposon transposition.

Genetic backgrounds of ES cells and EPSCs: AB2.2-129S5; E14-129P2; DR10 and DR25 were mixed 129S5 and C57BL/6 background. Other EPSC lines from eight-cell embryos or blastomeres were C57BL/6 background. Some EPSC lines for testing procedures had CD1 genetic background.

Cell lines from blastomeres or embryos were karyotyped at passages 12-15. For EPSCs, 4 out of 11 lines were karyotypically normal, whereas 3 out of 10 2i/LIF ES cells were normal.

\section{Derivation of EPSC from eight-cell embryos}

Each eight-cell embryo was transferred to one well of a 96-well SNL feeder plate or a gelatinized plate in EPSCM. Embryos were cultured in EPSCM for over 8 days until they hatched and formed outgrowths. The outgrowth was dissociated with Accutase or mechanically cut into small pieces with a mouth pipette and transferred into a new 96-well SNL feeder plate or a gelatinized plate in EPSCM. After 2-3 days, EPSC colonies reached $80-90 \%$ confluence, cells were passaged into 24-well SNL feeder plates or gelatinized plates, then expanded into six-well plates. EPSCs were frozen down in 90\% EPSCM + 10\% dimethylsulfoxide (DMSO) or 90\% FBS + 10\% DMSO and stored in liquid nitrogen. In general, using a SNL feeder layer, the EPSC derivation efficiency was close to $100 \%$, whereas only about $20 \%$ was achieved if embryos were on a gelatinized plate. In one experiment, from 24 eight-cell embryos cultured in $0.1 \%$ gelatin-coated wells, 5 EPSC lines were established. In another experiment, from four blastocysts cultured on SNL feeder, all formed outgrowths, one was accidently lost and three EPSC lines were established. The detailed systematic EPSC derivation protocol can be found at Protocol Exchange ${ }^{31}$.

\section{Preparing the embryos and foster recipients}

To produce embryos, CD1 females older than 8 weeks of age were super-ovulated by intraperitoneal injection of pregnant mare serum gonadotropin (Intervet, PMSG-Intervet), 7.5 international units each, followed $46-48 \mathrm{~h}$ later by injection of human chorionic gonadotropin (Intervet, Chorulon) 7.5 international units each, and were then mated to 
proven studs. Vaginal plugs were checked the following morning ( 0.5 d.p.c.). Eight-cell embryos and blastocysts were collected at 2.5 d.p.c. and 3.5 d.p.c. by flushing the oviduct and the uterus, respectively. Embryos were cultured in $1.0 \mathrm{ml} \mathrm{KSOM}$ medium (Millipore, MR-107-D) containing $3.0 \mu \mathrm{g} \mathrm{ml}^{-1}$ bovine serum albumin (BSA, Sigma, A3311) in an incubator with $5 \% \mathrm{CO}_{2}$ at $37^{\circ} \mathrm{C}$. CD1 females were mated with vasectomized C57BL6/ JCBA F1 males and used as recipients for embryo transfer at 2.5 d.p.c.

\section{Injection of ES cells and EPSCs}

ES cells or EPSCs were fed with fresh medium 3-4 h before they were harvested for injection. Injections were performed with a Nikon Eclipse TE2000-U inverted microscope unit. The micromanipulator was an Eppendorf InjectMan NI2 or Eppendorf TransferMan NK2. The outside diameter of the holding pipette was 95-105 $\mu \mathrm{m}$, the inside diameter 20-25 $\mu \mathrm{m}$; the outside diameter of the injection pipette was 18-20 $\mu \mathrm{m}$, the inside diameter 16-18 $\mu \mathrm{m}$. ES cell/EPSC microinjection was performed at room temperature on the inverted lid of a $100 \mathrm{~mm}$ Falcon culture dish in an injection drop of $1.0 \mathrm{ml} \mathrm{M} 2$ medium (Sigma, M7167) with $7.5 \mu \mathrm{g}$ cytochalasin B (Sigma, C6762). The embryo was injected with six to eight cells per eight-cell embryo for bulk injection or one cell per embryo for single-cell injection. After 45-50 embryos had been injected, they were washed three times in KSOM containing $3.0 \mathrm{mg} \mathrm{ml}^{-1}$ BSA. Embryos were then transferred into a well of a four-well plate containing $1.0 \mathrm{ml} \mathrm{KSOM}$. After overnight culture, around 20 injected embryos were transferred into both uterine horns of a 2.5 d.p.c. pseudopregnant CD1 mouse. The same microinjection protocol was used for single-cell injection, with one cell injected into the centre of an eightcell embryo. The contributions of mouse EPSCs to the trophectoderm, the ExE and placenta trophoblasts in chimaera embryos were reproduced in H.N.'s laboratory at the University of Tokyo.

\section{Whole-mount immunostaining of blastocysts and 5.5-6.5 d.p.c. embryos}

Immunostaining of early embryos was performed according to ref. ${ }^{32}$ with minor modifications. Blastocysts were washed in PBS/PVP (polyvinylpyrrolidone, $3 \mathrm{mg} \mathrm{ml}^{-1}$, Sigma, P5288), fixed in 2.5\% paraformaldehyde (PFA) for $15 \mathrm{~min}$ at room temperature, (5.5-6.5 d.p.c. embryos were fixed in 4\% PFA for $30 \mathrm{~min}$ ), permeabilized in PBS/PVP/ $0.025 \%$ Triton X-100 for $30 \mathrm{~min}$ (more than $2 \mathrm{~h}$ for 5.5-6.5 d.p.c. embryos), blocked in $\mathrm{PBS} / \mathrm{PVP} / 0.1 \% \mathrm{BSA} / 0.01 \%$ Tween-20/2\% donkey serum for $1 \mathrm{~h}$ or more at room temperature and incubated with primary antibodies in blocking solution at $4{ }^{\circ} \mathrm{C}$ overnight. Embryos were then washed with blocking solution three times for 15 min each, incubated with Alexa Fluor- or CF660C-conjugated secondary antibodies for $1 \mathrm{~h}$ at room temperature, washed with blocking solution three times for 15 min each, mounted in a small drop of Vectashield with DAPI (5.5-6.5 d.p.c. embryos went through 25, 50, 75 and 100\% Citifluor, mounted with Prolong antifade mountant with DAPI), covered with a coverslip and sealed with nail varnish.

For the embryo outgrowth, cells in a 96-well plate were fixed in 4\% PFA. The blocking solution was PBS/0.1\% Triton X-100/1\% BSA/3\% donkey serum, and the staining procedure was similar to that listed above. After secondary antibody incubation, cell nuclei were stained with DAPI and observed under a fluorescence microscope. This protocol was 
also used for immunostaining of cultured cells. Antibodies used are listed in Supplementary Table 7.

\section{Separation of blastomeres and derivation of EPSC lines in EPSCM}

Before separating the blastomeres, the four-cell to eight-cell embryos were cultured in 1.0 $\mathrm{ml} \mathrm{KSOM}$ containing $3.0 \mathrm{mg} \mathrm{ml}^{-1}$ BSA and $7.5 \mu \mathrm{g}$ cytochalasin B (Sigma, C6762) for at least half an hour. The embryos were suspended in a $100 \mu$ l Tyrode's solution (Sigma, T1788) to wash away KSOM media, and then transferred into and suspended in a new 100 $\mu \mathrm{l}$ Tyrode's solution drop. The embryos were transferred into a $100 \mu \mathrm{l}$ M2 drop immediately after the zona pellucida dissolved in Tyrode's solution. The embryos were washed a few times in M2 drops. The embryos were passed up and down several times through a blunt needle (inner diameter $40 \mu \mathrm{m}$ ). The individual blastomeres could then be collected for the next experiment. To derive EPSC lines from a single blastomere, individual blastomeres were plated into a 96-well plate on an SNL feeder (one blastomere per well). The progress of blastomeres was monitored daily. At around day 7, some small cell clusters formed and the cells continued to proliferate to form EPSC colony-like outgrowths; at day 12, colonies were picked and dissociated with Accutase, and transferred into new 96-well plates on SNL feeder. The cells were further expanded in 24-well and 6-well plates. EPSCs were frozen down for storage in liquid nitrogen or maintained in EPSCM for further analysis. From 32 blastomeres cultured on SNL feeder, 9 EPSC lines were established.

\section{Minimal requirement of inhibitors in acquiring the expanded potential}

AB2.2 ES cells with mCherry reporter were cultured in KSR-2i/LIF medium, supplied with A-149259, XAV939, JNK inhibitor III, SB203580 individually or A-149259+XAV939; or in KSR-LIF they were supplied with CHIR99021, A-419259 and XAV939; alternatively, AB2.2 EPSCs were cultured in EPSCM minus CHIR99021, A-419259 or XAV939, respectively. Cells were cultured in media for at least five passages on SNL feeder. The night before microinjection, the medium was changed to M15; the next morning, cells were fed with fresh M15 at least $1 \mathrm{~h}$ before microinjection. Cells were dissociated into single cells with Accutase. Normally six to eight cells were injected into one eight-cell embryo; after injection, embryos were cultured in M15 in uncoated four-well plates or low attachment sixwell plates for $72 \mathrm{~h}$ at $37{ }^{\circ} \mathrm{C}, 5 \% \mathrm{CO}_{2}$. The embryos were fixed in $2.5 \%$ PFA and immunostained with mCherry and $\mathrm{Cdx} 2$ antibody with the above-mentioned protocol. The contribution of mCherry ${ }^{+}$cells in trophectoderm and inner cell mass were scored. The cells cultured in KSR-2i/LIF/A-419529 were further characterized for gene expression profile by RT-qPCR and their in vivo contribution analysed by flow cytometry of 14.5 d.p.c. placenta and trophoblast markers in sorted mCherry ${ }^{+}$cells; 14.5 d.p.c. yolk sac sections were immunostained with mCherry antibody to detect their contribution in extra-embryonic endoderm.

\section{Teratoma formation}

Mouse EPSCs were re-suspended in PBS, and $5 \times 10^{6}$ EPSCs were injected subcutaneously into both dorsal flanks of 8-week-old male NSG mice (NOD.Cg-Prkdcscid Il2rgtm1 Wj1/ SzJ, The Jackson Laboratory). When the size of the teratomas reached $1.2 \mathrm{~mm}^{2}$, they were 
dissected, fixed overnight in 10\% phosphate-buffered formalin and embedded in paraffin before sectioning.

\section{Embryo dissection, imaging, placenta cell dissociation, FACS analysis and immunostaining}

Embryos (14.5 d.p.c.) were dissected and imaged using a Leica M205FA Automated Fluorescence Stereo Microscope (100 ms for phase and $5 \mathrm{~s}$ for mCherry). Placenta, yolk sac and embryo samples from mCherry ${ }^{+}$chimaeras were taken for genotyping. Dissected placentae were cut into fragments of approximately $1.0 \mathrm{~mm}$ diameter on ice and digested with $2.35 \mathrm{ml}$ placenta digestion solution (5\% FBS, $10 \mathrm{mM}$ HEPES, $100 \mu \mathrm{l}$ enzyme D, $50 \mu \mathrm{l}$ enzyme R and $12.5 \mu$ l enzyme A in Hank's balanced salt solution according to the Lamina Propria Dissociation Kit instructions) for $30 \mathrm{~min}$ at $37^{\circ} \mathrm{C}$ with shaking. After digestion, the placentae were dissociated into single cells by pipetting vigorously and then filtered through a $70 \mu \mathrm{m}$ cell strainer (BD Biosciences). Single cells from placentae were treated with ACK Lysing Buffer (BioWhittaker) to remove the red blood cells and re-suspended in FACS buffer (BD Pharmingen) for FACS analysis or sorting. Fetal livers and fetal brains (14.5 d.p.c.) were dissociated in Hank's balanced salt solution by pipetting vigorously and filtering through a $70 \mu \mathrm{m}$ cell strainer. All FACS analysis was done on a BD LSRFortessa Cell Analyzer (BD Biosciences). Placenta cells were sorted on MoFlo XDP (Beckman Coulter).

Cells sorted by flow cytometry were enriched on polylysine-coated slides by cytospin at 500 r.p.m., 3 min, fixed in 4\% PFA; immunostaining was performed with the same protocol as for cultured cells. Antibodies used are listed in Supplementary Table 7.

\section{Placental tissue and yolk sac section immunostaining}

Paraffin-embedded 14.5 d.p.c. placenta and yolk sac sections were de-paraffinized in xylene and $100 \%, 95 \%, 80 \%, 70 \%$ and $50 \%$ ethanol for 3 min each and rinsed with running cold water. Antigens were retrieved in sodium citrate buffer ( $10 \mathrm{mM}$ sodium citrate, $0.05 \%$ Tween-20, pH 6.0) by microwaving for $15 \mathrm{~min}$, followed by rinsing with tap water. The slides were then washed in PBS/0.025\% Triton X-100 (PBST) twice for 5 min each, blocked in PBST/1.0\% BSA/5.0\% donkey serum for $1 \mathrm{~h}$ at room temperature, briefly rinsed with PBST and incubated with anti-mCherry, anti-GFP antibody (Abcam) at $4{ }^{\circ} \mathrm{C}$ overnight. The slides were washed with PBST three times for 15 min each, blocked for at least 15 min, incubated with Alexa Fluor (Life Technologies) or CF660C- (Biotium) conjugated secondary antibody for $1 \mathrm{~h}$ at room temperature, washed with PBST three times for $15 \mathrm{~min}$ each. The sections were mounted in a small drop of Vectashield with DAPI, covered with a coverslip and sealed with nail varnish. The sections were observed and imaged with a fluorescence microscope. For mCherry and Tfap2c co-staining, to reduce autofluorescence, slides were irradiated with ultraviolet light for $2 \mathrm{~h}$ before de-paraffining, and treated with Sudan Black for 20 min after blocking. Antibodies used are listed in Supplementary Table 7. The technical details of reducing background staining have been published ${ }^{33}$. 


\section{TSC derivation and culture}

Cdx2-GFP-EPSCs were cultured on gelatinized plates for three passages to deplete residual SNL feeders, and were plated at $1 \times 10^{4}$ cells per well into six-well plates with mouse embryonic fibroblasts (MEFs) in TX medium29 with slight modifications: insulin, holotransferrin and sodium selenite were replaced with N2 (Invitrogen) used at 1:200. At day 3, cells were dissociated with $0.025 \%$ trypsin/EDTA and re-suspended in PBS/0.1\% BSA for flow cytometry sorting. Cdx2-GFP ${ }^{+}$cells were sorted and plated in a well of six-well plates with MEFs and TX medium. After 7 days, TSC-like colonies emerged and single colonies were picked into 24-well plates with MEFs and TX medium for expansion and characterization. From ten colonies picked, five EPSC TSC lines were established.

\section{TSC transplantation}

Five million TSCs or EPSC TSC were re-suspended in $200 \mu \mathrm{l}$ TS medium with FGF4 (R\&D, catalogue number 235-F4-025/CF, lot RAF-0313071) ${ }^{34}$ and were injected subcutaneously into 8-week-old female NSG mice. After 7 days, lesions were dissected, documented and fixed in 4\% PFA, and embedded in paraffin for sectioning. Sections were stained with haematoxylin and eosin or subjected to immunofluorescence with the aforementioned protocol.

\section{TSC injection for chimaera embryos}

TSCs or EPSC TSCs cultured in TX medium were switched to TS medium overnight before injection. The injection was performed in M2 with 10\% FCS; 15-20 cells were injected into $\mathrm{CD} 1$ eight-cell embryos or blastocysts. The injected embryos were cultured in KSOM with $10 \% \mathrm{FCS}$ at $37{ }^{\circ} \mathrm{C}, 5 \% \mathrm{CO}_{2}$ overnight or $1-2 \mathrm{~h}$ for eight-cell embryos or blastocysts, respectively, before being transferred into the uterine horns of 2.5 d.p.c. pseudopregnant CD-1 females. Chimaera embryos were dissected 4 days after transfer for analysis (6.5 d.p.c.). For in vitro observation and immunostaining of blastocysts, embryos were further cultured in KSOM with 10\% FCS for $48 \mathrm{~h}$ and embryos were fixed.

\section{XEN cell line derivation from ES cells or EPSCs}

We used the XEN cell derivation protocol developed in ref. 30 with slight modifications. Gata6H2B-Venus EPSCs ${ }^{35,36}$ were cultured on gelatin-coated six-well plates for more than three passages to remove residual SNL feeder. Ninety thousand cells were seeded on gelatincoated six-well plates in standard XEN cell medium (RPMI1640, plus 15\% FBS); 24 h later, the medium was switched to cXEN derivation medium (standard XEN medium supplied with $0.01 \mu \mathrm{M}$ all trans-retinoic acid and $10 \mathrm{ng} \mathrm{ml}^{-1}$ activin A). After 2 days, the cells were dissociated with trypsin and plated at 1:1 ratio into MEF-coated six-well plates with standard XEN medium. The medium was changed every 2 days. After about 5 days, XEN cell-like cells emerged; 7 days later, Gata6H2B-Venus ${ }^{+}$XEN cell colonies were picked and dissociated with trypsin, and cells were plated onto new MEF-coated six-well in standard XEN cell medium. The EPSC-derived XEN cells were continuously passaged on MEFs for characterization. From 12 colonies picked, 8 XEN cell lines were established.

XEN cells were injected into recipient embryos for chimaeras following the same procedure as described for TSC injection. The cells derived from the donor EPSC-XEN cells were 
identified by Venus expression (expressing from the Gata6 locus, thus of the donor cell origin) and staining of Gata6 (labelling all PrE derivatives) in the chimaeras.

RT-qPCR

Total RNA was isolated using an RNeasy Mini Kit (Qiagen) for cultured cells or RNeasy Micro Kit (Qiagen) for sorted placental cells. RNA was subsequently quantified and treated with gDNA WipeOut to remove genomic DNA. Complementary DNA (cDNA) was prepared using a QuantiTect Reverse Transcription Kit (Qiagen). TaqMan Gene Expression Assays (Life Technologies) (Supplementary Table 4) and ABsolute Blue qPCR ROX Mix (ABgene) were used for probe-based qPCR assays. SYBR Green ROX qPCR Mastermix (Qiagen) was used to check the expression of trophoblast-specific genes in sorted placental cells. Primer pairs are listed in Supplementary Table 5. All qPCR reactions were performed on ABI 7900 HT Sequence Detection System (Life Technologies). Gene expression was determined relative to Gapdh or $A c t b$ using the $\Delta C_{\mathrm{t}}$ method. Data are shown as the mean and s.d.

\section{DNA genotyping}

AB2.2 mCherry EPSCs were injected into ROSA26-GFP-SB10 blastocysts. The chimaeras were collected at 14.5 d.p.c. $\mathrm{mCherry}^{+}$and $\mathrm{mCherry}^{-}$placenta cells were sorted, from which genomic DNA was extracted for PCR to amplify $m$ Cherry and SB10. Amplification of a region in the Oct4 distal enhancer region served as the genomic DNA quality and PCR control. PCR primers are listed in Supplementary Table 6.

\section{DMR analysis}

Bisulfite treatment was performed using the EpiTect Bisulfite Kit (Qiagen) according to the manufacturer's recommendations. Genomic DNA PCR for Elf5 promoter regions was performed using primer pairs described before ${ }^{37}$. PCR products were cloned into pGEM-T Easy Vector and sequenced from both ends.

\section{Kinase inhibition and western blot}

A total of $1.0 \times 10^{6}$ ES cells were seeded into gelatinized wells of a six-well plate in one of the following media: M15, N2B27-2i/LIF, KSR-2i/LIF or EPSCM. After 48 h, whole-cell protein was extracted from harvested cells and quantified using a BCA assay (Thermo Scientific). Total protein $(40 \mu \mathrm{g})$ was fractioned on a 4-12\% Bis-Tris Novex gel (Invitrogen), electroblotted onto PVDF membranes, and probed with the following antibodies: pERK, ERK, pSrc, Src, p-P38, P38, pJNK, JNK and axin1 (all from Cell Signaling). a-Tubulin (Abcam) was used as the loading control. Blots were incubated with horseradish peroxidasecoupled anti-rabbit or anti-mouse IgG and developed with ECL Prime (Amersham). Antibodies used are listed in Supplementary Table 7.

\section{Wnt signalling detection}

To investigate Wnt signalling in EPSCs, $1.0 \times 10^{6}$ cells were seeded in M15, N2B27-2i/LIF, KSR-2i/LIF and EPSCM for $48 \mathrm{~h}$. Cytoplasmic and nuclear proteins were extracted with a NE-PER Nuclear and Cytoplasmic Extraction Kit (Thermo Scientific) for western blot to 
detect phospho- $\beta$-catenin (Cell Signaling), $\beta$-catenin (Sigma) and active- $\beta$-catenin (Millipore). $a$-Tubulin (Abcam) and histone $\mathrm{H} 3$ were used as loading controls for cytoplasmic and nuclear protein, respectively. For the TOPflash assay, $2.0 \times 10^{6}$ cells were co-transfected with $20 \mu \mathrm{g}$ TOPflash and $2 \mu \mathrm{g}$ pRL-TK by Nucleofection (Amaxa). Cells were split 1:9 into a 24-well plate in M15and N2B27-2i/LIF and EPSCM for $48 \mathrm{~h}$. Cell lysates were prepared for luciferase assay. Antibodies used are listed in Supplementary Table 7.

\section{LIF response in ES cells and EPSCs}

For LIF stimulation, $1.0 \times 10^{6}$ cells were seeded in EPSCM for $24 \mathrm{~h}$. Cells were washed with PBS and cultured in DMEM/F12/KSR for $4 \mathrm{~h}$. LIF was then added at 0.0, 0.5, 1.0, 5.0 and $10.0 \mathrm{ng} \mathrm{ml}^{-1}$ for $30 \mathrm{~min}$. Whole-cell protein was extracted for western blot using pSTAT3 (Cell Signaling) and STAT3 (BD Biosciences) antibodies. a-Tubulin (Abcam) was used as the loading control. Antibodies used are listed in Supplementary Table 7.

To analyse the expression of downstream target genes of JAK-STAT3 and MAPK signalling at the transcriptional level, both E14Tg2a ES cells and EPSCs were deprived of LIF for $4 \mathrm{~h}$, as above. LIF at $1.0 \mathrm{ng} \mathrm{ml}^{-1}$ was then added back for $1 \mathrm{~h}$. Total RNA was extracted and reverse transcribed to make cDNA. qPCR was performed using TaqMan Gene Expression Assays (ABI) (Supplementary Table 4).

\section{Reprogramming MEFs}

To reprogram MEFs, episomal vectors with Oct4, c-Myc, Klf4 and Sox 2(OCKS 4F, $5 \mu \mathrm{g}$ ) and Rarg, Lrh1 (RL 2F, $5.0 \mu \mathrm{g}$ ) were first mixed with $1.0 \times 106$ cells in OptiMEM (Invitrogen). The cells were electroporated with Amaxa Nucleofector (Lonza, Germany, programme MEF A-023). After electroporation, the cells were plated onto 10-cm dishes with SNL feeders in M15 for $24 \mathrm{~h}$. The medium was changed every 2 days. At day 7, colonies emerged, and at day 14 the medium was switched to EPSCM for an additional 6 days. Colonies were picked into 24 wells on SNL feeders in EPSCM for expansion and characterization.

\section{Overexpressing axin1 in ES cells}

Mouse Axin1 cDNA was amplified from MSCVhyg-F-Axin1 (a gift from K. Ge, Addgene plasmid 24928) ${ }^{38}$, cloned into pCR Blunt II TOPO vector (Life Technologies, catalogue number 45-0245) and verified by sequencing. Axin1 cDNA was then linked in frame with $m$ Cherry by T2A sequence and cloned in the piggyBac transposon under the control of CAG promoter or doxycycline inducible promoter. For transfection, $1.0 \times 10^{6} \mathrm{Cdx} 2-\mathrm{GFP}$ ES cells were electroporated with $5 \mu \mathrm{g}$ PBCAGAxin1-2A-mCherry and $2 \mu \mathrm{g}$ transposase; or $5 \mu \mathrm{g}$ PBTET Axin1-2A-mCherry, $2 \mu \mathrm{g}$ PBEF1a TET3G and $2 \mu \mathrm{g}$ Transponsase by Amaxa Nucleofector (programme: mES cell A-023). After transfection, cells were seeded in sixwell plates on SNL feeder cultured with M15 medium; the medium was refreshed $24 \mathrm{~h}$ later (for inducible axin 1 expression, $2 \mu \mathrm{g} \mathrm{ml}^{-1}$ doxycycline was added into the medium) and cultured for another 2 days; cells were dissociated with Accutase and mCherry ${ }^{+}$cells were sorted by MoFlo XDP cell sorter (Beckman Coulter). The mCherry ${ }^{+}$cells were cultured on SNL feeder with KSR-2i/LIF medium for three passages, then transfected with $5 \mu \mathrm{g}$ 
PBPGK $H 2 B$-Venus and $2 \mu \mathrm{g}$ transposase per $1.0 \times 10^{6}$ cells under the same transfection program. Three days after transfection, mCherry $^{+}$and Venus ${ }^{+}$cells were sorted and cultured on SNL feeder with KSR-2i/LIF for more than three passages before introduction into eightcell embryos for in vivo assay.

\section{Single-cell RNA-seq library preparation}

The preparation was previously described ${ }^{39}$. In brief, 5,000 trypsin-dissociated mouse EPSCs (DR10) were loaded onto the 10-17 $\mu \mathrm{m}$ C1 Single-Cell Auto Prep IFCs (Fluidigm), and cell capture was performed according to the manufacturer's protocol. The individual capture site was inspected under light microscopy to confirm the presence of a single cell. The locations of empty capture sites and sites containing multiple cells or burst cells were noted for downstream quality control during data analysis. The lysis and reverse transcription mixes were then prepared with a SMARTer PCR cDNA Synthesis kit (Clontech) and an Advantage 2 PCR kit (Clontech) according to Fluidigm recommendations. A total of $1.0 \mu$ of the ERCC Spike-In Control Mix (Ambion) at a 1:400 dilution was added to the lysis mix to allow control of the technical variation of the library preparation protocol. Reverse transcription and PCR were performed, and cDNA was harvested by a C1 SingleCell Auto Prep system according to the manufacturer's settings. The success of cDNA preparation was confirmed by the optimal DNA signal detected by a 2100 Bioanalyzer with a high-sensitivity DNA chip (Agilent). Multiplex sequencing libraries were prepared using a Nextera XT DNA Sample Preparation Kit and a Nextera Index Kit (Illumina) according to the recommendation in the $\mathrm{C} 1$ Single-Cell Auto Prep manual. The libraries of individual cells were then pooled and sequenced on four lanes of HiSeq 2500 (Illumina) to generate 100-bp paired-end reads through the Sanger Institute in-house DNA sequencing pipeline.

\section{Read alignment and quality control of dataset}

Pair-end reads from both bulk and single-cell RNA-seq datasets were aligned to the Mus musculus (GRCm38.74) genome by STAR (version STAR_2.3.0e_r291) with specific additional parameter settings: '-outFilterMultimapScoreRange 1-outSAMstrandField intronMotif ${ }^{40}$. The quantification of gene expression was performed by the htseq-count module from the HTSeq package (http://www-huber.embl.de/users/anders/HTSeq/) with gene annotation from GTF files (GRCm38.74 for mouse) with parameter '-s no' in union mode ${ }^{41}$. The count matrix of individual cells was normalized by size factors with DESeq2 (ref. ${ }^{42}$ ). As a form of quality control in the single-cell data set, cells with fewer than 500,000 counts in annotated features, expressing fewer than 4,000 genes or a high percentage (>10\%) of counts mapping to mitochondria-encoded genes were removed from subsequent analysis.

\section{Identification of highly variable genes in EPSCs}

Highly variable genes were identified by testing the observed gene expression variability against the observed spike-in variability following the method described in ref. ${ }^{39}$. Genes with Benjamini-Hochberg-adjusted $P$ values less than 0.1 were classified as highly variable and selected for Gene Ontology term enrichment analysis. 


\section{Unsupervised hierarchical clustering of RNA-seq data and box plots comparing the expression variability}

The heatmap shown in Fig. 3a represents the inter-sample correlation coefficient calculated by the Spearman's rank correlation method. Low expression genes with normalized counts less than 5 were excluded.

A Wilcoxon rank-sum test was performed to compare the expression variability, measured as the squared coefficient of variation of putative genes in EPSCs compared with M15 ES cells $(P<0.001)$. In Fig. 3b, the bar in the boxes, the upper and lower hinges, and the upper and lower whiskers mark the median, the first and third quartiles, and the smallest and largest observations excluding outliers, respectively. Outliers were determined as observations less than the first quartile $-1.5 \times$ interquartile range and observations larger than the third quartile $+1.5 \times$ interquartile range.

\section{Differential gene expression analysis}

Differential expression analysis was performed with DESeq2 (ref. ${ }^{42}$ ). Cooks distance testing was turned off to accommodate the noisy expression nature of single-cell data, and counts from the ERCC spikes in the EPSCM data sets were removed from the input matrix. Differential expression with an adjusted $P<0.05$ and $\mid \log _{2}$ (fold change) $\mid>1$ were considered significantly differentially expressed.

\section{Gene Ontology enrichment analysis}

Gene Ontology term enrichment analysis was done with DAVID ${ }^{43}$ (http://

david.abcc.ncifcrf.gov/). Gene symbols were used as the input gene list, and the mouse genome was chosen as the background. Terms were defined as significantly enriched if the adjusted $P$ value (Benjamini-Hochberg method) was less than 0.05 .

\section{Gene set enrichment analysis}

Gene set enrichment analysis (Fig. 3d) was performed with the javaGSEA application (version 2.1.0; http://www.broadinstitute.org/gsea/downloads.jsp) with the 'weighted' enrichment statistics setting ${ }^{44}$. The mouse pre-implantation embryonic stage-enriched gene sets were compiled by analysing the published expression count matrix from ref. 23 . The top 500 genes with higher average expression than other stages after size factor normalization by DESeq2 were considered stage-enriched and used as the testing gene set. The normalized count matrices of the EPSC and standard 2i/LIF ES cell single-cell data sets were used as input expression data sets. Enrichment was considered significant if the FDR was below 0.1 and the nominal $P$ value was below 0.05 .

\section{Estimating the pre-implantation embryonic developmental time frame of EPSCs}

The embryo (from the zygote to 16 -cell embryos) raw count matrix from ref. ${ }^{44}$ was first merged with the ES count matrix of EPSCs and 2i/LIF ES cells and normalized by DESeq2 size factors. Only genes found to be differentially expressed between EPSCs and 2i/LIF ES cells were included for later analysis. The normalized embryo count matrix was then transformed by $\log _{2}($ nCount +1$)$ and PCA performed by the prcomp () function from the 
stats package in R. The scores were then used to predict the scores of EPSCs and 2i/LIF ES cells by the predict() function from the stats package in $\mathrm{R}$. The three-dimensional plot was generated by the scatterplot3d function from the scatterplot3d package in $\mathrm{R}$.

\section{Comparison of EPSCs, Hhex-Venus+ ES cells, MERV-TdTomato+ ES cells and in vivo iPS cells}

The raw RNA-seq data from in vivo reprogrammed iPS cells (iviPS1-6) from ref. 26, HhexVenus $^{+}$ES cells from ref. 19 (both 2i/LIF and unsorted 2i/LIF) and MERV-TdTomato ${ }^{+}$ES cells from ref. 25 were downloaded from GSE48364, GSE45182 and GSE33923, respectively, and aligned to GRCm38 mouse genome by STAR. Gene expression was then quantified as described above by HTSeq-count. To normalize the difference between studies and sequencing platforms, the expression data were first normalized by DESeq2 size factor and quantified as relative expression to the mean expression value of 2i/LIF ES cells. Similarly, Hex-Venus ${ }^{+}$ES cells were normalized to unsorted 2i/LIF ES cells from the same study. PCA was performed on $\log _{2}$-transformed expression data by the prcomp() function from the stats package in R. To quantify the expression of endogenous retroviral transcripts in EPSCs, sequences of repeat elements (both mouse and ancestor-shared) were downloaded from http://www.girinst.org/repbase/index.html. A custom GTF file and genome index were then constructed by STAR with individual repeat class treated as individual chromosome. Reads were then realigned to the new index. Expression was quantified by HTSeq-count as described above and normalized by size factors computed by DESeq2. The relative expressions of EPSCs and MERV-Tdtomato ${ }^{+}$ES cells were then calculated relative to that of MERV-TdTomato ${ }^{-}$ES cells.

\section{ChIP and analysis}

The H3K4me3, H3K27me3, H3K27ac and input ChIP library of EPSCs (DR10) were prepared on the basis of a modified ChIP protocol from ref. ${ }^{45}$. In brief, about 20 million cells were cross-linked with $1.1 \%$ formaldehyde for $10 \mathrm{~min}$ at room temperature. Crosslinking was then quenched with $0.125 \mathrm{M}$ glycine. Cell pellets were washed with PBS, snap frozen in liquid nitrogen and stored at $-80{ }^{\circ} \mathrm{C}$ until further processing. The chromatins were sheared by Bioruptor Pico (Diagenode) for $5 \mathrm{~min}$ in $30 \mathrm{~s} / 30 \mathrm{~s}$ on/off cycles.

Immunoprecipitation was performed with $2.5 \mu \mathrm{g}$ antibodies pre-washed and pre-attached to protein A Dynaebeads (Life Technologies) overnight at $4{ }^{\circ} \mathrm{C}$. H3K4me3, H3K27me3, $\mathrm{H} 3 \mathrm{~K} 27 \mathrm{ac}$ antibodies are listed in Supplementary Table 7 . The beads were then washed and cross-linking was reversed with the elution buffer at $65^{\circ} \mathrm{C}$ for $4 \mathrm{~h}$. Immunoprecipitated DNAs were purified by proteinase K digestion and with a Qiagen miniElut kit (Qiagen). The multiplex sequencing libraries were prepared with the microplex library construction kit (Diagenode) following the manufacturer's instructions. The DNAs were amplified for 11 cycles and the quality of the library was checked on a Bioanalyzer (Agilent) using a highsensitivity DNA kit. The library was sequenced on two lanes of a HiSeq2500 in multiplex.

The single-end 50-bp reads were then mapped to the mouse reference genome GRCm38 by the Burrows-Wheeler Aligner ${ }^{46}$ (bwa-0.5.10) according to the in-house Sanger DNA pipeline. The H3K4me3 and H3K27me3 ChIP-seq data of TNGA mouse ES cells cultured in 2i/LIF and a TNGA gnomic DNA control were downloaded from the National Center for 
Biotechnology Information (NCBI) Gene Expression Omnibus SuperSeries GSE23943. H3K27ac ChIP-seq data of R1 mouse ES cells cultured in 2i/LIF were downloaded from GSE56138 (ref. ${ }^{47}$ ). The data were remapped to the GRCm38 mouse genome by Bowtie2 (ref. ${ }^{48}$ ) with default parameters. Read depth between data sets was then normalized by read shuffling and random sampling to 20,664,503 reads (H3K27me3) and 10,311,175 reads (H3K4me3). H3K4me3 and H3K27me3 enriched peaks were detected by MACS (version 2.1.0) with the flag '--broad' turned on for detection of broad histone modification peaks. The signal tracks were generated by MACS (version 1.4.2) 49 and visualized by the University of California, Santa Cruz (UCSC) genome browser. H3K4me3 and H3K27ac signal tracks of oocytes, two-cell embryos and eight-cell embryos were downloaded from GSE72784 (refs $28,{ }^{50}$ ). Peak coordinates were then intersected with the gene promoter windows ( $\pm 1 \mathrm{~kb}$ from TSS) by Bedtools ${ }^{51}$ to identify H3K4me3- and H3K27me3-associated genes. Bivalent genes were defined as genes that were both H3K4me3- and H3K27me3associated. Comparisons of $\mathrm{H} 3 \mathrm{~K} 4 \mathrm{me} 3$ and $\mathrm{H} 3 \mathrm{~K} 27 \mathrm{me} 3$ signals at the extended promoter window (TSS $\pm 3 \mathrm{~kb}$ ) and gene body were done by ngs.plot (version 2.41.3) ${ }^{52}$. To compare the H3K4me3 and H3K27me3 signal distribution at genes with different expression levels, expressed genes of EPSCs were first ranked on the basis of their average length corrected for mean-normalized count level, and genes in the first, second, third and fourth quartiles were classified into four groups as 'very high', 'high', 'low' and 'very low'. Signal plots across the gene body were then generated by ngs.plot. Length correction was performed by dividing the normalized count by union exon length (in kilobases). Bivalent domainassociated genes of four-cell and eight-cell embryos were retrieved from the supplementary data of ref. ${ }^{50}$.

We used the 'findpeak -style super' module of HOMER version 4.8.3 to identify H3K27ac super-enhancer regions in 2i/LIF ES cells and EPSCs ${ }^{53}$. Peak regions with superEnhancer slope $>1$ were classified as super-enhancers. The super-enhancer regions of EPSCs and ES cells were intersected with TSS $\pm 1 \mathrm{~kb}$ regions using bedtools to identify super-enhancerassociated genes. Genes that were common between replicates were used for later EPSC-ES cell comparison.

\section{Mass spectrometry}

Genomic DNA was quantified using PicoGreen assay (Invitrogen), digested using DNA Degradase Plus (Zymo Research) for $90 \mathrm{~min}$ at $37{ }^{\circ} \mathrm{C}$ and analysed by liquid chromatography-tandem mass spectrometry on an LTQ Orbitrap Velos mass spectrometer (Thermo Scientific) fitted with a nanoelectrospray ion source (Proxeon, Odense, Denmark). Mass spectral data for cytosine, $5 \mathrm{mC}$ and $5 \mathrm{hmC}$ were acquired as previously described ${ }^{45}$. The $5 \mathrm{mC}$ and $5 \mathrm{hmC}$ levels were normalized to total cytosine levels. Error bars represent s.d. of at least three technical replicates per sample.

\section{Digital microscopy imaging}

Immunofluorescence-stained samples were examined with a Leica DM5000B microscope equipped with narrow band-pass filters for FITC, Cy3.5, Cy5.5 and DAPI fluorescence. Images were captured with a monochrome digital camera (ORCA-03G, Hamamatsu) and processed with the SmartCapture software (Digital Scientific UK). 


\section{Confocal microscopy}

Images were recorded by using an inverted 780 Zeiss laser scanning microscope with $20 \times$ or $40 \times$ objectives. The lasers were set to $405 \mathrm{~nm}$ and optimized for collection of emitted light in the 410-489 $\mathrm{nm}$ band for DAPI, and an excitation wavelength of $561 \mathrm{~nm}$ and optimized for collection of emitted light in the 551-638 nm band for AlexFluor594. The stacks of $Z$ sections were recorded at $0.8-1.0 \mu \mathrm{m}$ intervals to encompass the full depth of embryos. Images were processed with ZEN software (Zeiss).

\section{Time-lapse imaging of embryo development in EPSCM}

Transgenic Oct4-GFP four-cell to eight-cell embryos were cultured in a gelatinized 96-well plate in EPSCM. At $80 \mathrm{~h}$ in EPSCM, the embryos were imaged on a Leica AF6000 fluorescence microscope. The microscope stage and objectives were enclosed by a cage incubator and maintained at $37{ }^{\circ} \mathrm{C}, 5 \% \mathrm{CO}_{2}$. A $20 \times$ objective was used for all positions. Time-lapses were acquired using a GFP filter-set and transmitted light images were sequentially captured. Images were acquired at $30 \mathrm{~min}$ intervals for $55 \mathrm{~h}$ in total. The image sequence was exported as an .avi file.

\section{Statistical analysis}

No statistical methods were used to predetermine sample size. The experiments were not randomized. The investigators were not blinded to allocation during experiments and outcome assessment. Experimental data are shown as mean and s.d. All statistical analyses were either conducted with Prism (GraphPad) or are specified in the relevant sections. 


\section{Extended Data}

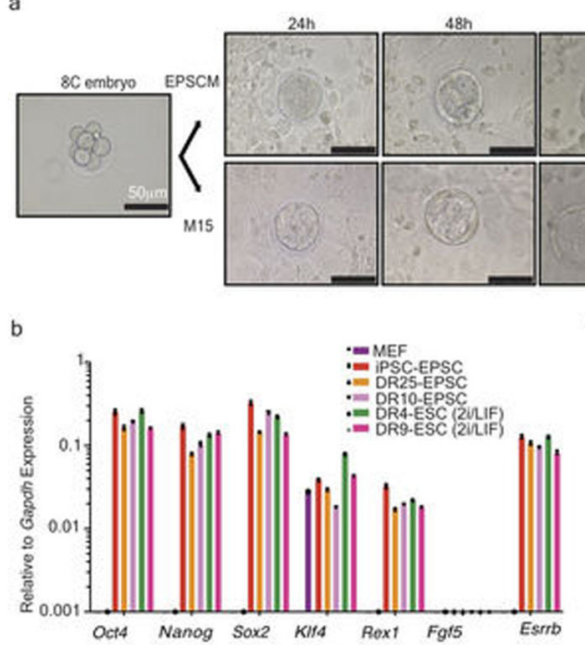

$72 \mathrm{~h}$
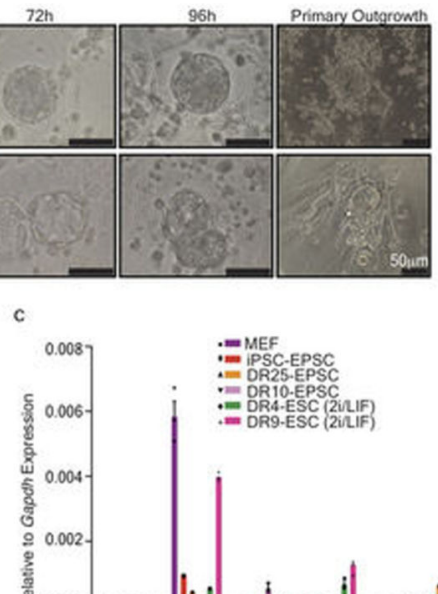
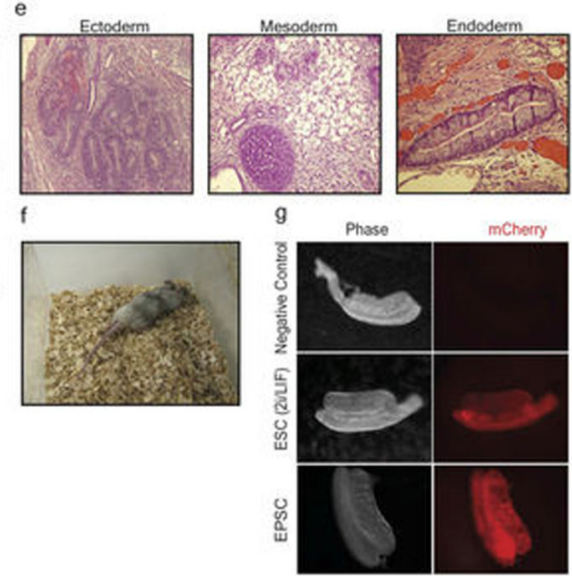
d

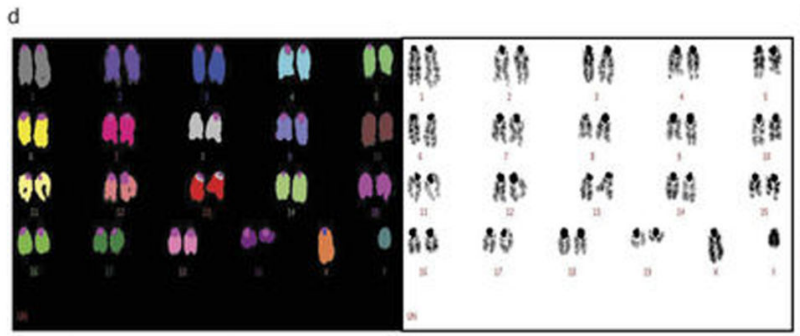

j
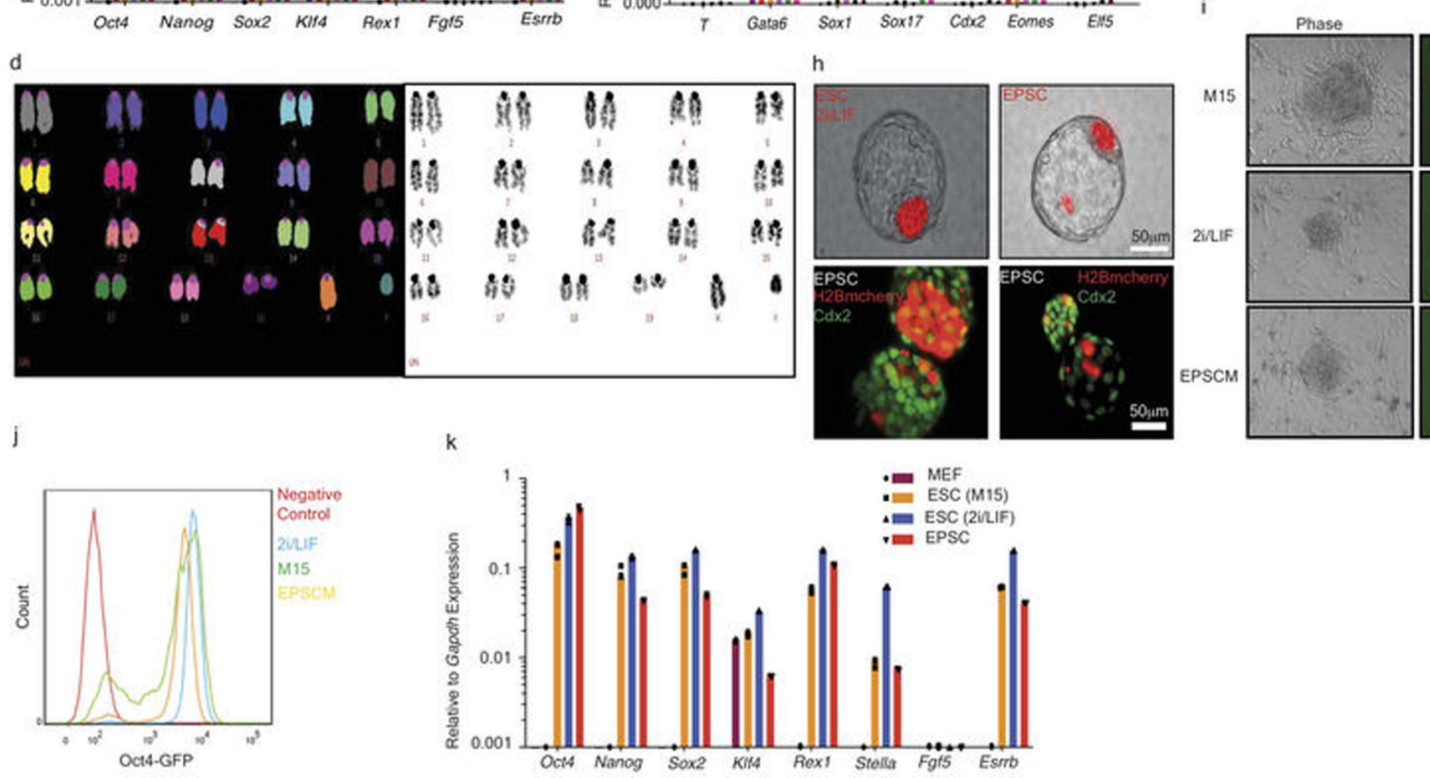

Rex1-GFP
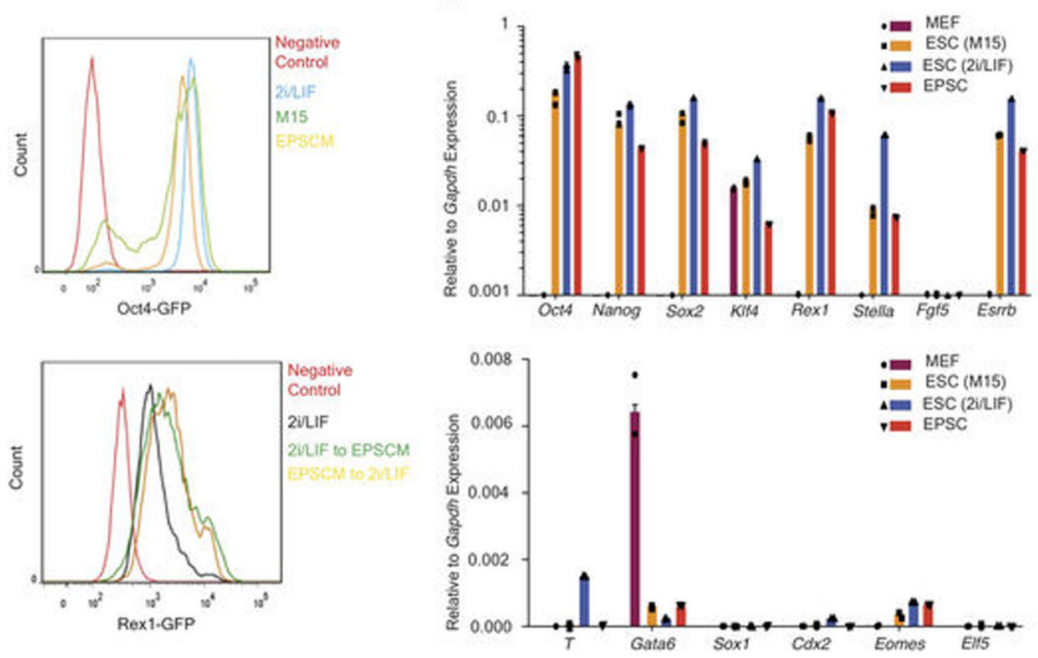

Ext Data Fig 1.

a, Phase images showing development of eight-cell mouse embryos in EPSCM or in M15. Note that in embryos in EPSCM, the blastocoel cavity was filled with cells and the embryos hatched at day 7 or 8 . b, EPSCs derived from pre-implantation embryos express pluripotency markers. Analysis of pluripotency markers in two embryo-derived EPSC lines (DR25 and DR10), one reprogrammed iPS cell-EPSC line and two embryo-derived 2i/LIF 
ES cell lines (DR4 and DR9). Expression of pluripotency genes was relative to Gapdh in the RT-qPCR assay. Data are mean \pm s.d. $(n=3)$. Overlaid dot plots indicate the distribution of the data. $\mathbf{c}$, Expression of lineage-specific genes in the cells described in b. Expression of these genes was relative to Gapdh. Data are mean \pm s.d. $(n=3)$. Overlaid dot plots indicate the distribution of the data. d, Spectral karyotyping of DR10-EPSCs (normal karyotype in eight out of ten metaphases) at passage 10. e, Mature teratomas from DR10-EPSCs. Left: ectodermal neural tube-like structures; middle: mesodermal cartilage; right: an endodermal gland structure of gastrointestinal type and mesodermal muscle fibres. The sections were stained with haematoxylin-eosin. f, A chimaera derived from DR10-EPSCs. g, The mCherry ${ }^{+}$EPSCs show extensive contribution to the 14.5 d.p.c. gonad, similar to 2i/LIF ES cells. h, Additional images of EPSC contributions in the trophectoderm of the blastocyst. The two images on the top are merged live images (phase and mCherry), which show the contribution of mCherry (cytoplasmic)-labelled 2i/LIF ES cells or ES cell-EPSCs. The two at the bottom are immunostaining images to detect H2B-mCherry-expressing AB2.2 ES cell-EPSCs and the trophectoderm cells $\left(\mathrm{Cdx}^{+}\right)$. i, Rex 1-GFP reporter ES cell colonies in M15, 2i/LIF or EPSCM on SNL feeder. $\mathbf{j}$, Expression of the reporter GFP in ES cells cultured in EPSCM (passage 3). Top: Oct4-GFPES cells (E14Tg2a) cultured in M15, 2i/LIF or EPSCM. In all conditions, Oct4-GFP expression was detected by FACS. Bottom: Rex1-GFPES cells (AB2.2 background) have comparable levels of Rex 1 expression (GFP) when cultured in EPSCM or subsequently returned to 2i/LIF. Negative control: wild-type ES cells. $\mathbf{k}$, Expression of pluripotency genes or lineage-specific genes in ES cells cultured in EPSCM (ES cell-EPSCs). Expression of these genes was relative to Gapdh. Data are mean \pm s.d. ( $n$ $=2$ ). Overlaid dot plots indicate the distribution of the data. 
a

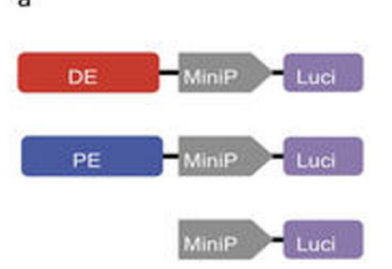

b
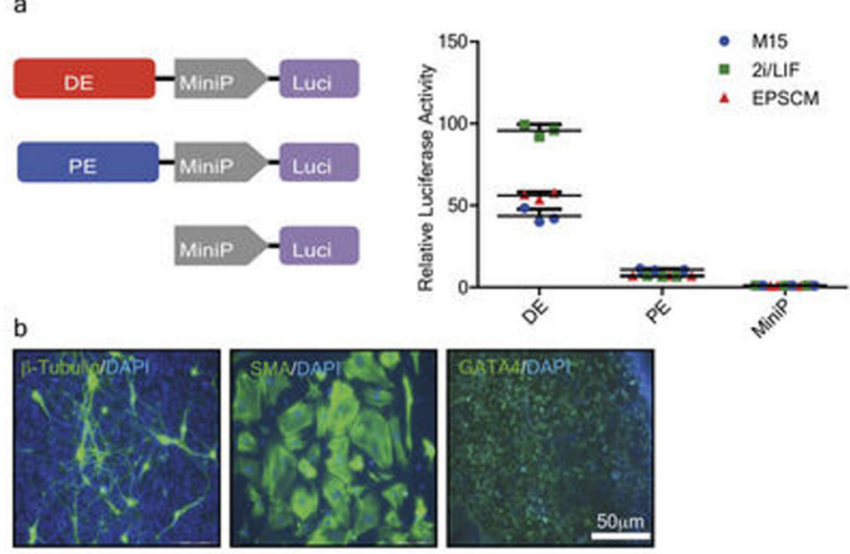

c
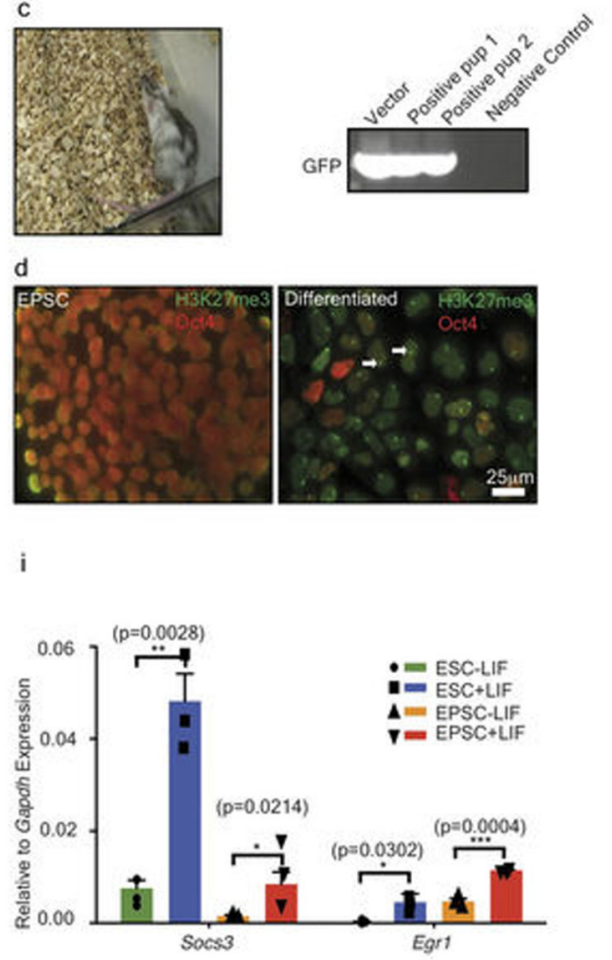

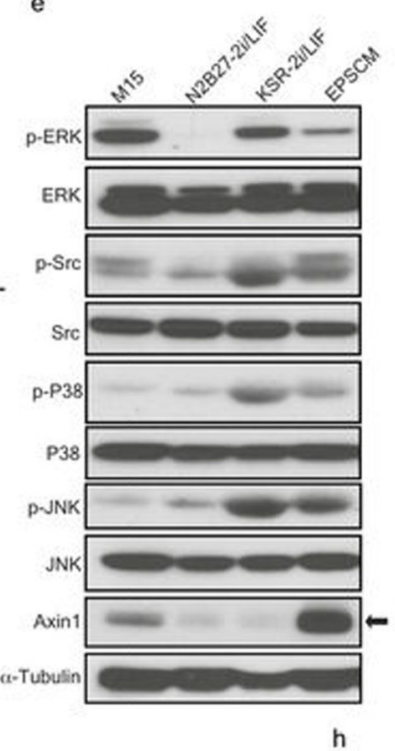

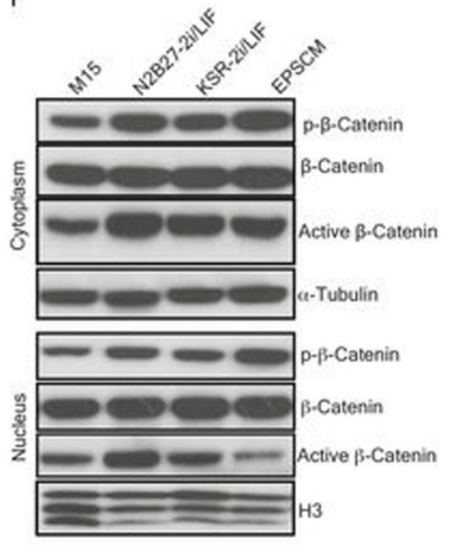

g
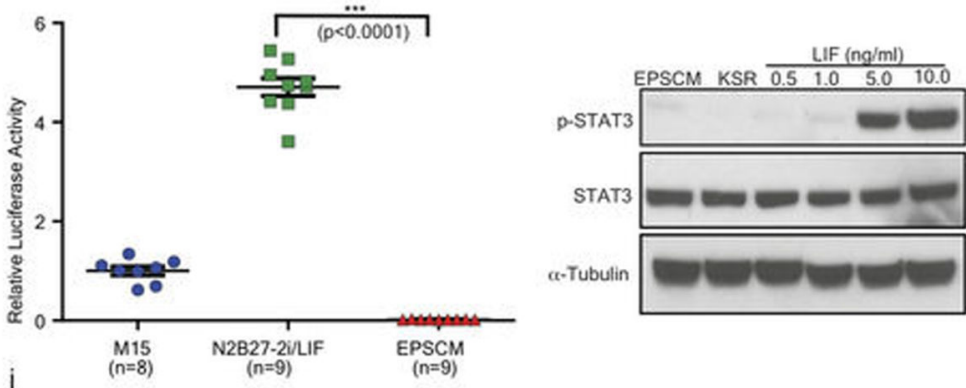

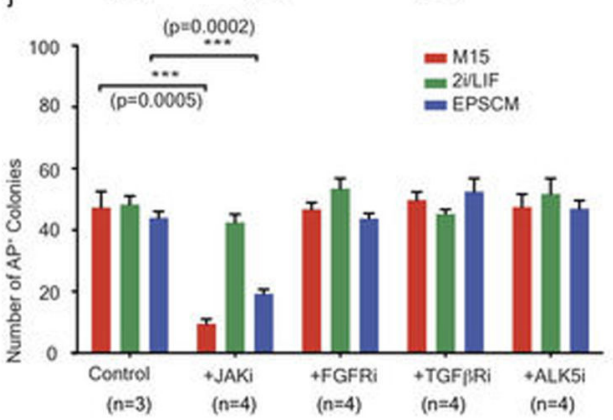

Ext Data Fig 2.

a, Left: Oct4 distal and proximal enhancer luciferase reporter constructs. DE, the Oct 4 distal enhancer; PE, the Oct4 proximal enhancer; MiniP, the minimum promoter. The Oct 4 distal enhancer is active in ES cells cultured in EPSCM. Right: luciferase activities of the Oct4 DE and PE constructs were normalized to that of the MiniP construct in the same cell type. Data are mean \pm s.d. $(n=3)$. $\mathbf{b}$, Differentiation of ES cell-EPSCs in vitro into cells of three germ layers detected by the immunostaining of class III $\beta$-tubulin, $a$-smooth muscle actin (SMA) and Gata4. c, A male chimaera from ES cell-EPSCs, and germline transmission of the Rex1-GFP allele from the chimaera. $\mathbf{d}$, Activation of X chromosomes in female ES cellEPSCs. EPSCs or the cells differentiated from EPSCs were co-immunostained for H3K27me3 and Oct4. No discrete H3K27me3 foci were found in EPSCs. Once the cells 
differentiated, the foci appeared in almost all cells. Arrows point to H3K27me3 foci. e, f, Effects of small-molecule inhibitors on their respective targets. p-ERK, phosphorylated ERK; p-Src, phosphorylated Src; p-P38, phosphorylated P38; p-JNK, phosphorylated JNK. a-Tubulin was used as the loading control. Note the substantial increase of axin1. EPSCM contains $20 \% \mathrm{KSR}$ so KSR-2i/LIF is the appropriate negative control. XAV939 in EPSCM stabilizes axin1 by inhibiting its ubiquitination. EPSCs had considerably elevated levels of axin 1 compared with controls (arrow), which caused accumulation of phosphorylated $\beta$ catenin in both the cytoplasm and nuclei and decreased active $\beta$-catenin in the nucleus. $a$ Tubulin and histone $\mathrm{H} 3$ were used as the loading controls of cytoplasmic and nuclear proteins, respectively. The gel source data are in Supplementary Fig. 1. g, Reduced $\beta$ catenin-LEF/TCF activity in EPSCs detected by TOPflash luciferase assay. TOPflash luciferase activity was normalized to that of EPSCs grown in M15 medium. Data are mean \pm s.d. ( $n=8$ for M15, 9 for N2B27-2i/LIF and EPSCM). Two-sided Student's $t$-test was performed. $* * * P<0.001$. h, Western blot analysis shows increased p-STAT3 in ES cellEPSCs with increased concentrations of LIF. The gel source data are in Supplementary Fig. 1. i, Upregulation of downstream LIF pathway genes in ES cell-EPSCs in response to LIF stimulation. Two-sided Student's $t$-test was performed. Data are mean \pm s.d. $(n=3)$. ${ }^{*} P<$ $0.05 ; * * P<0.01 ; * * * P<0.001$. j, Signalling pathway dependence in ES cell-EPSCs. ES cells and EPSCs ( $n=100$ for each cell type) cultured in M15, 2i/LIF or EPSCM with inhibitors of JAK (JAK inhibitor 1), FGFR (SU5402), TGFßR (A83-01) or ALK5 (SB505124). Alkaline phosphatase-positive $\left(\mathrm{AP}^{+}\right)$colonies were scored after 10 days. The JAK inhibitor substantially reduced the number of $\mathrm{AP}^{+}$colonies from these cells. By contrast, ES cells cultured in EPSCM did not appear to be sensitive to FGFR or TGF $\beta$ R inhibitors, similar to 2i/LIF ES cells. Data are mean \pm s.d. $(n=3$ for control, 4 for inhibitors). $* * * P<0.001$. 
a
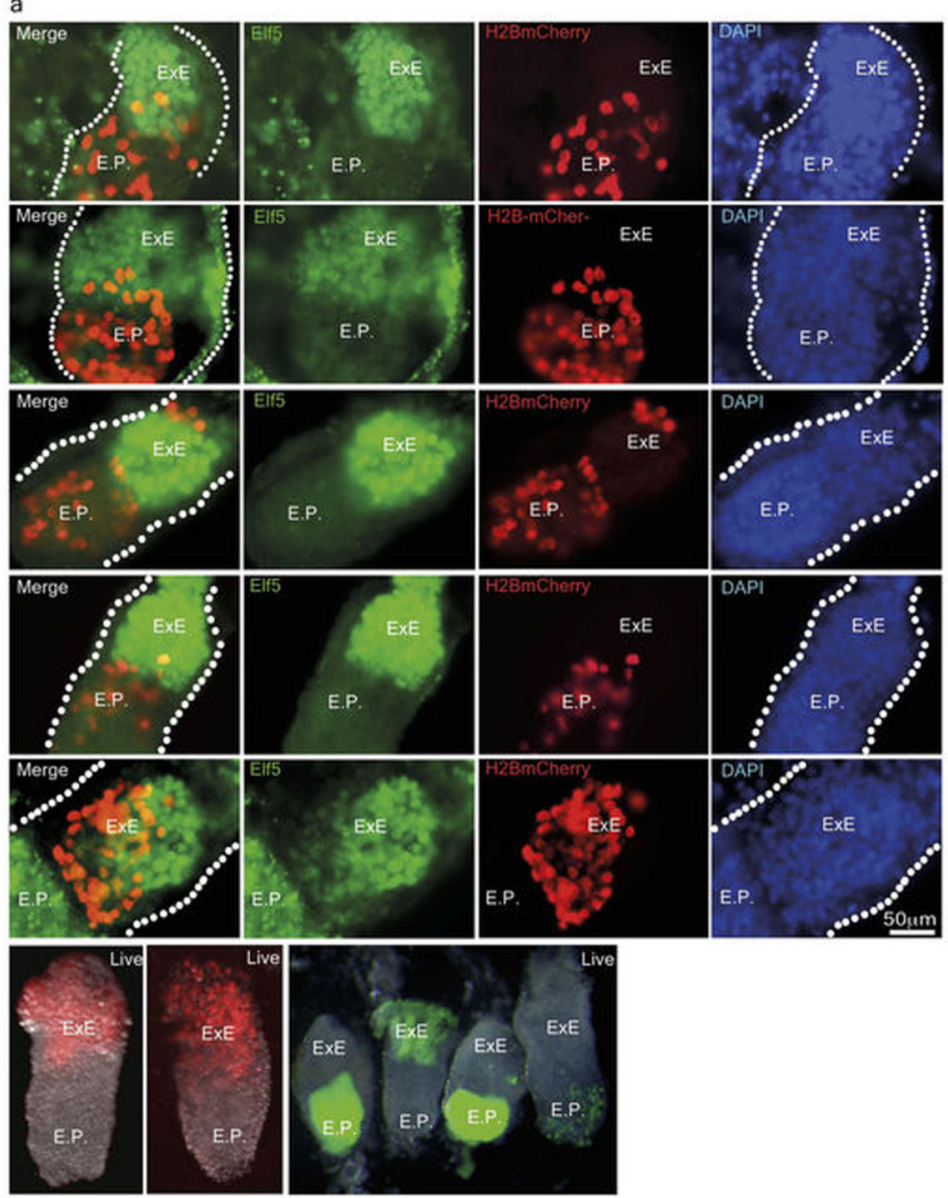

b

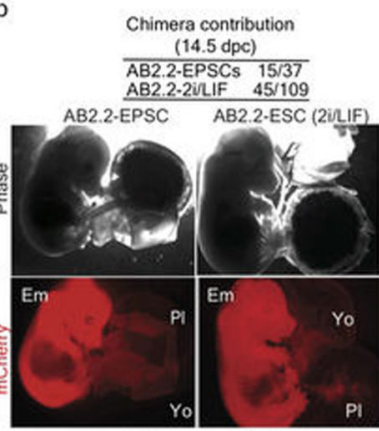

Chimera contribution $\frac{(14.5 \mathrm{dpc})}{0 / 10}$ Negative control 0/10
DR10.EPSCs $31 / 58$
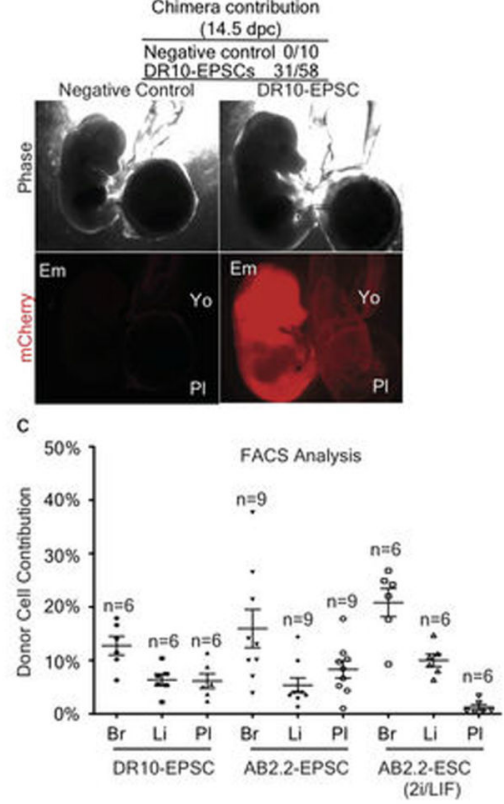

d
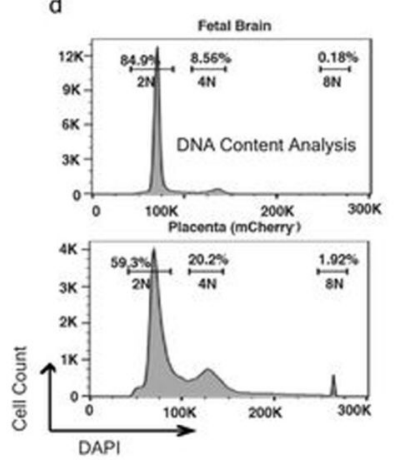

e
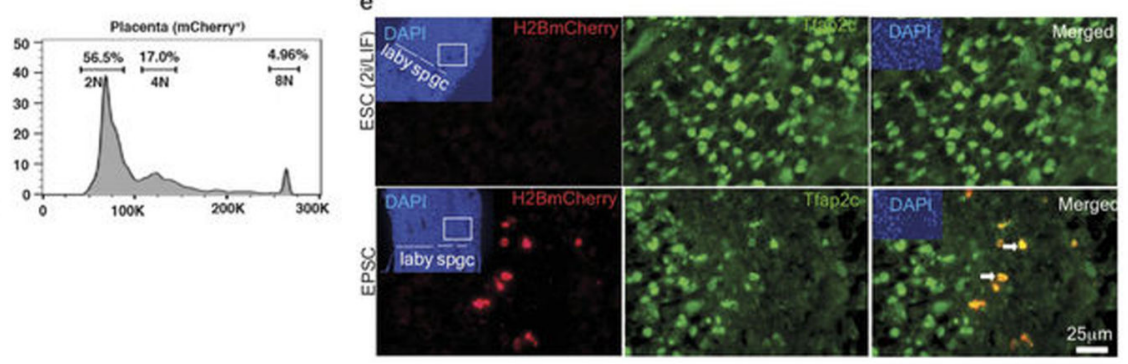

Ext Data Fig 3.

a, Whole-mount immunostaining of 6.5 d.p.c. chimaeras for H2B-mCherry and Elf5. In the chimaeras shown, the descendants of EPSCs were found both in the embryo proper and in the ExE. One of the chimaeras (number five from the top) has EPSC contribution predominantly in the Elf-5-expressing ExE. The live images at the bottom show the contribution of EPSCs predominantly in the ExE and ectoplacental cone (EPC) (mCherry in Cambridge, GFP in Tokyo). b, Whole-mount fluorescence imaging of 14.5 d.p.c. chimaeras from morulas injected with ES cells or EPSCs. Negative control: wild-type embryo or 
chimaeras from unlabelled EPSCs. Em, embryo; Yo, yolk sac; Pl, placenta. Inset tables show the frequency of contribution by the stem cells to the chimaeras. $\mathbf{c}$, Percentages of EPSC or ES cell contributions in independent chimaeras ( $n=6$ for DR10-EPSC; $n=9$ for AB2.2EPSC; $n=6$ for AB2.2-ES cell control). Cells from the brain (Br), liver (Li) and placenta (Pl) were collected for flow cytometric analysis. d, Detection of polyploid placenta cells by flow cytometry. A distinct population of $8 N$ cells is present in both $\mathrm{mCherry}^{+}$and mCherry ${ }^{-}$ placenta cells from an AB2.2-EPSC chimaera. Fetal brain cells were used as the control. No mCherry ${ }^{+} 8 N$ cells could be detected in the placenta from $2 \mathrm{i} /$ LIF ES cells. e. Placenta sections of 14.5 d.p.c. chimaeras from either ES cells or EPSCs $\left(\mathrm{AB} 2.2, \mathrm{mCherry}^{+}\right)$were coimmunostained for mCherry and Tfap2c. mCherry ${ }^{+}$cells were detected in the EPSC chimaera placenta; some of these cells were positively stained for both mCherry and Tfap2c (arrows). The areas framed in the low-magnification DAPI staining (insets) indicate the positions of the placenta section images. Abbreviations: laby, labyrinth; sp., spongiotrophoblast layer; gc, trophoblast giant cell layer.

a

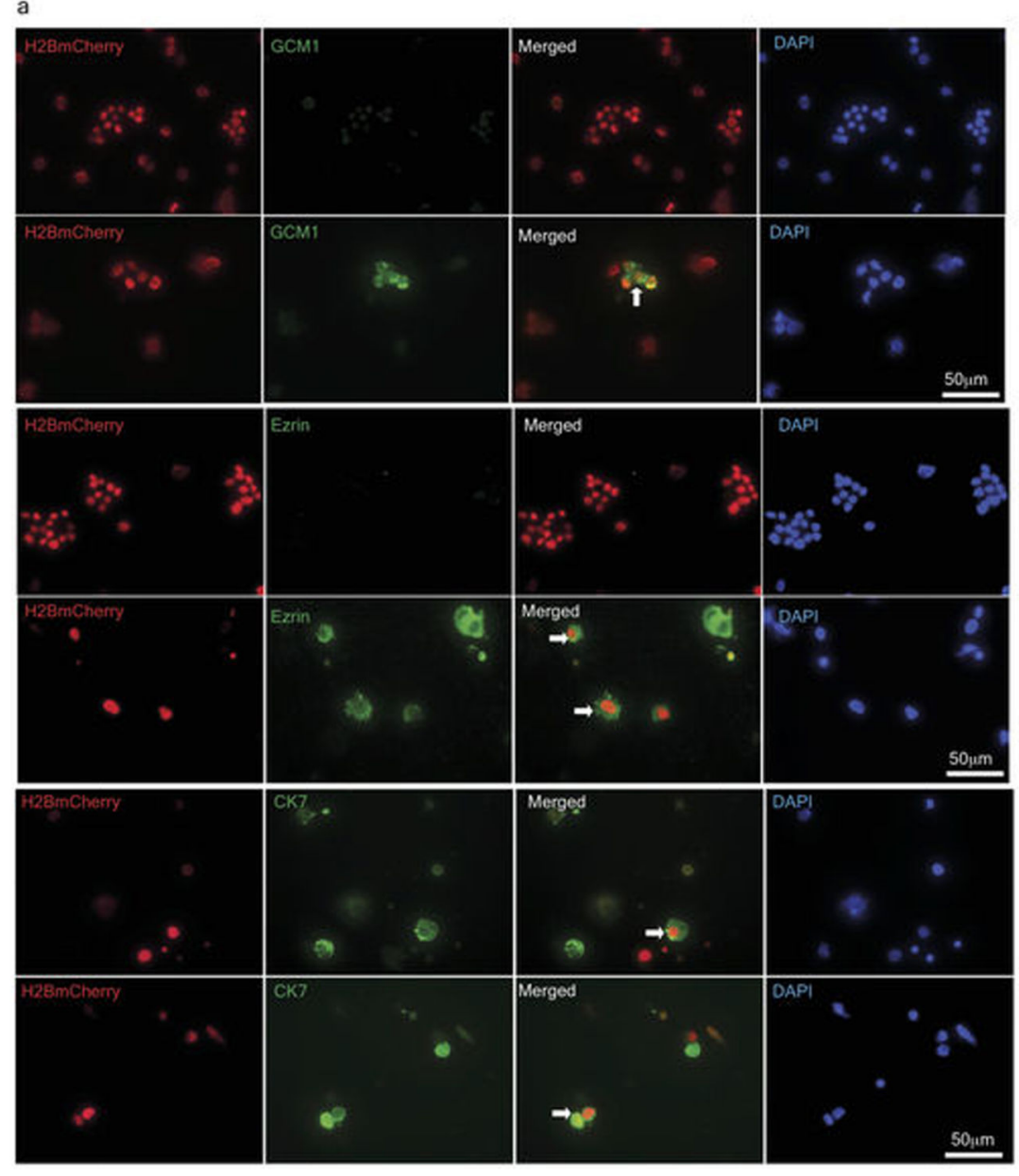

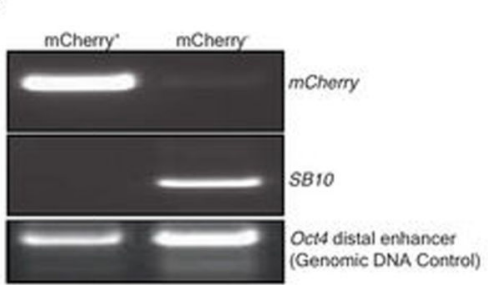

C
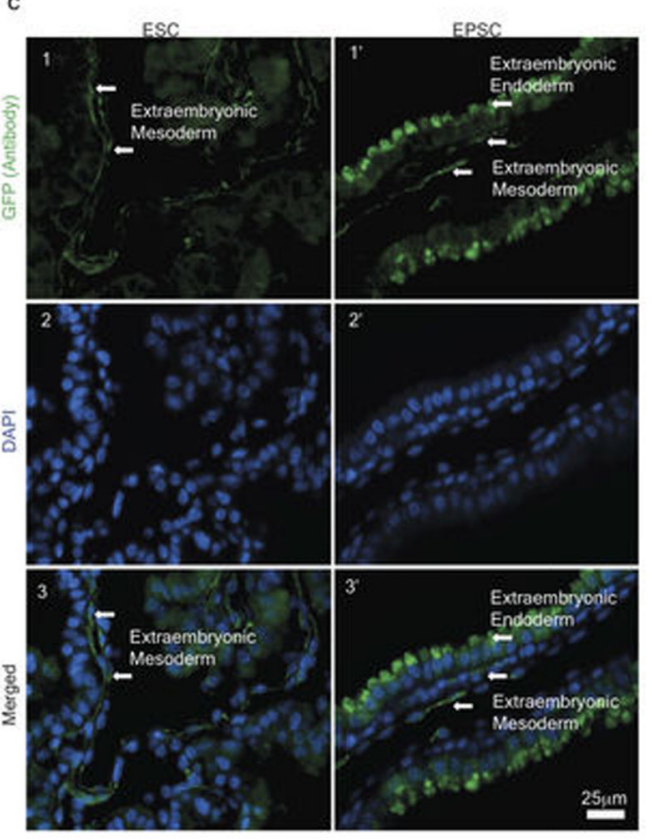

Ext Data Fig 4.

a, Whole-mount fluorescence of 14.5 d.p.c. chimaeras from eight-cell embryos and a single EPSC or ES cell. Negative control: wild-type embryo. Inset table shows the frequency of 
contribution by the stem cells to the chimaeras. b, Flow cytometry analysis of mCherry ${ }^{+}$ placenta cells in single-EPSC-injected chimaeras. c, Expression of trophoblast genes in mCherry $^{+}$and mCherry ${ }^{-}$placenta cells sorted from the single-EPSC chimaera. Expression was relative to $A c t b$ and normalized to fetal brain expression. Data are mean \pm s.d. $(n=3)$. Overlaid dot plots indicate the distribution of the data. Sorting was performed as in Fig. 2c. d, Detection of polyploid placenta cells. A distinct population of $8 N$ cells was found both in mCherry ${ }^{+}$and in mCherry ${ }^{-}$placenta cells in an EPSC chimaera.

a

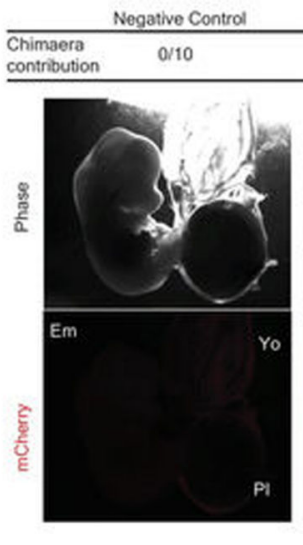

c

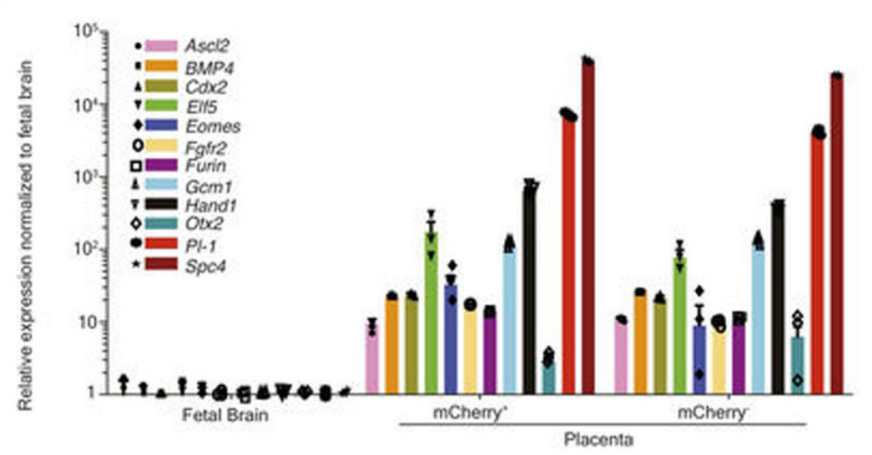

b

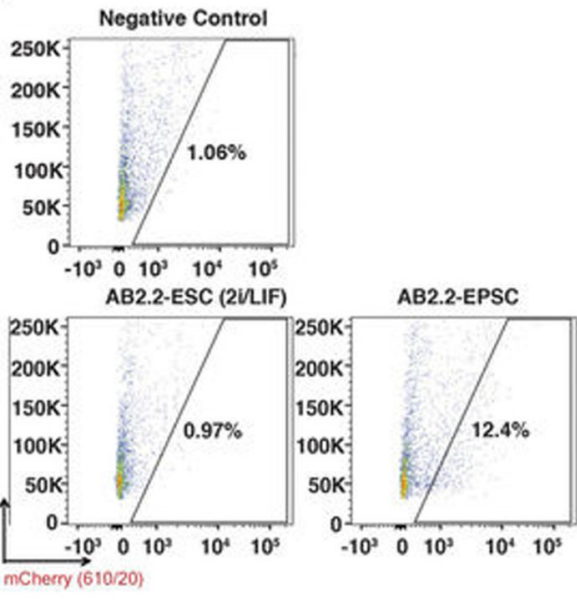

DNA Content Analysis
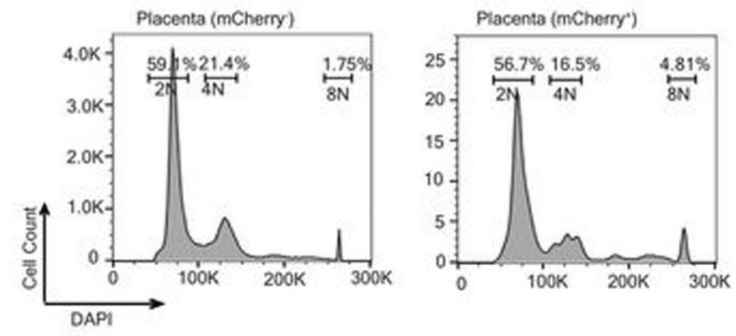

Ext data Fig 5.

a, Whole-mount fluorescence of 14.5 d.p.c. chimaeras from eight-cell embryos and a single EPSC or ES cell. Negative control: wild-type embryo. Inset table shows the frequency of contribution by the stem cells to the chimaeras. b, Flow cytometry analysis of mCherry ${ }^{+}$ placenta cells in single-EPSC-injected chimaeras. c, Expression of trophoblast genes in mCherry $^{+}$and mCherry ${ }^{-}$placenta cells sorted from the single-EPSC chimaera. Expression was relative to $A c t b$ and normalized to fetal brain expression. Data are mean \pm s.d. $(n=3)$. Overlaid dot plots indicate the distribution of the data. Sorting was performed as in Fig. 2c. $\mathrm{d}$, Detection of polyploid placenta cells. A distinct population of $8 N$ cells was found both in $\mathrm{mCherry}^{+}$and in $\mathrm{mCherry}^{-}$placenta cells in an EPSC chimaera. 
a

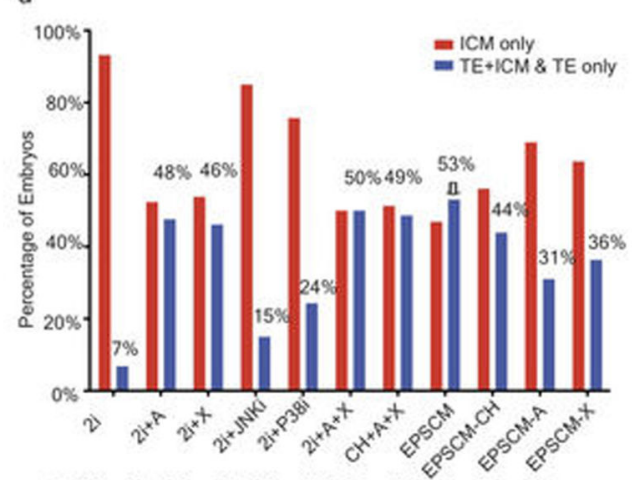

c

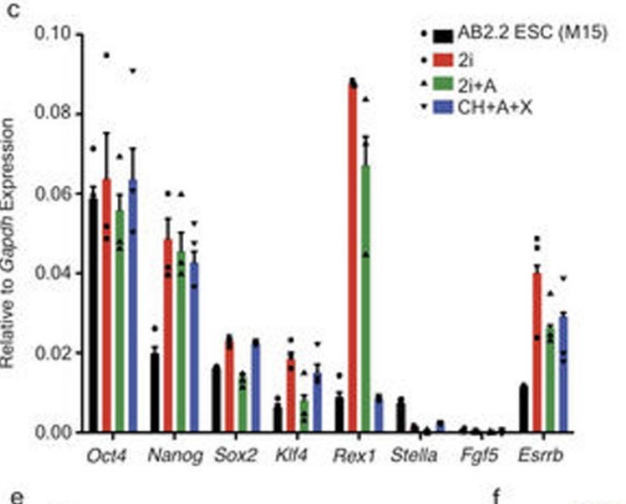

e

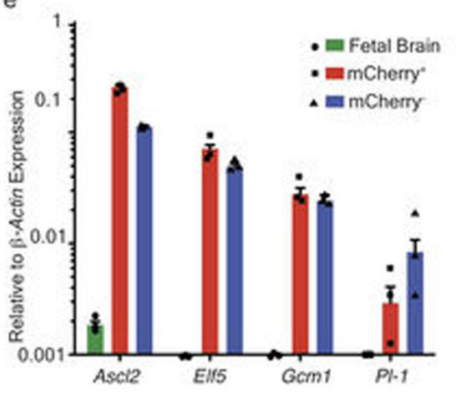

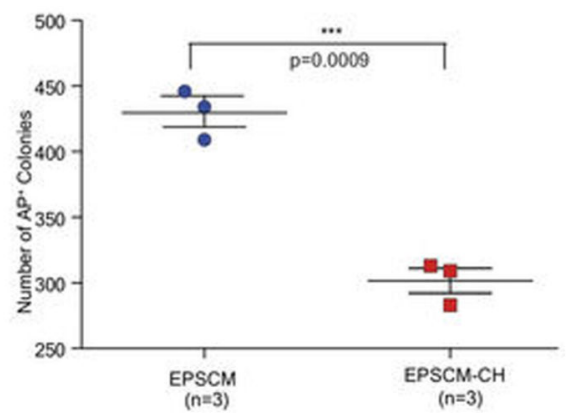
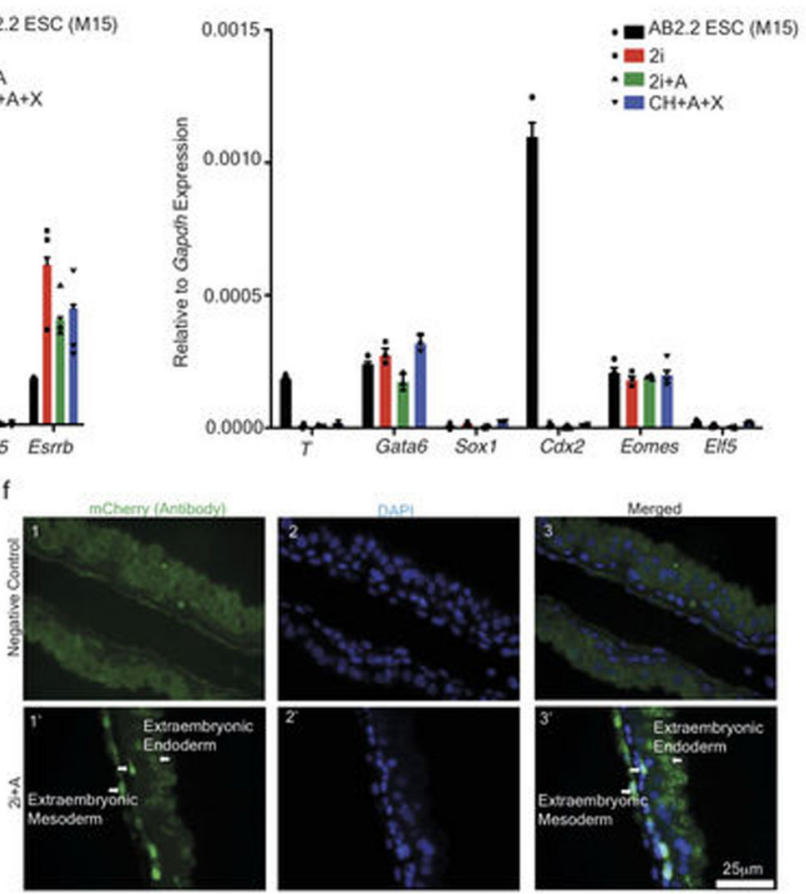
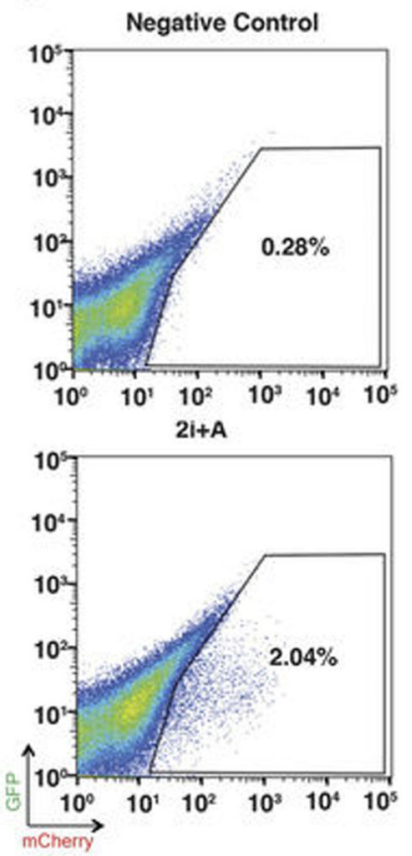

Ext Data Fig 6.

a, Effects of individual inhibitors on trophectoderm contribution. Mouse ES cells (mCherry ${ }^{+}$ AB2.2) were cultured in various combinations of inhibitors for at least five passages before injection into eight-cell embryos and examination at the blastocyst stage. Blastocysts were scored for the donor cells' trophectoderm contribution. A, A419259; X, XAV939; JNKi, JNK Inhibitor VIII; p38i, SB203580; CH, CHIR99021. All culture conditions contained KSR and LIF. For each culture condition, 38-58 embryos were scored. b, The effect of CHIR99021 (CH) in EPSCM on cells. One thousand EPSCs cultured in either EPSCM or EPSCM minus $\mathrm{CH}$ were plated for single-cell colony formation assay. $\mathrm{AP}^{+}$colonies were scored on day $7(n=3)$. A two-sided Student's $t$-test was performed. Data are mean \pm s.d. ( $n$ $=3)$. $* * * P<0.001$. c, Gene expression in ES cells cultured in minimum sets of inhibitors. Expression of genes was relative to Gapdh in the RT-qPCR assay. Data are mean \pm s.d. $(n=$ 3). Overlaid dot plots indicate the distribution of the data. d, Flow cytometry analysis of $\mathrm{mCherry}^{+}$placenta cells of 14.5 d.p.c. chimaeras from ES cells cultured in $2 \mathrm{i}+\mathrm{A}$ medium. To 
minimize residual background cell sorting, a GFP channel was used to exclude autofluorescence. e, Expression of trophoblast-enriched genes in cells sorted from the placenta of chimaeras. The mCherry ${ }^{+}$placenta cells were sorted as in $\mathbf{d}$ from chimaeras of ES cells cultured in $2 \mathrm{i}+\mathrm{A}$ medium. Expression was relative to $A c t b$. Data are mean \pm s.d. ( $n$ $=3$ ). Overlaid dot plots indicate the distribution of the data. f, Yolk sac sections of 14.5 d.p.c. chimaeras of ES cells ( $2 \mathrm{i}+\mathrm{A})$ or the wild-type negative control. Immunostaining and imaging were performed in the same way as in Extended Data Fig. 4c. The mCherry fluorescence signal was quenched by fixation. Donor cells were stained by a CF660Cconjugated antibody against mCherry (panels 1 and $1^{\prime}$ ). DAPI staining was used to detect the nucleus (panels 2 and $2^{\prime}$ ). Donor-cell derivatives were found in both the extra-embryonic mesoderm (endothelial and mesothelial cells) and extra-embryonic endoderm cells in the yolk sac of EPSC chimaera, but not in the yolk sac of the negative control chimaera ( 3 and 3 '). Arrows point to extra-embryonic endoderm, extra-embryonic mesoderm. 


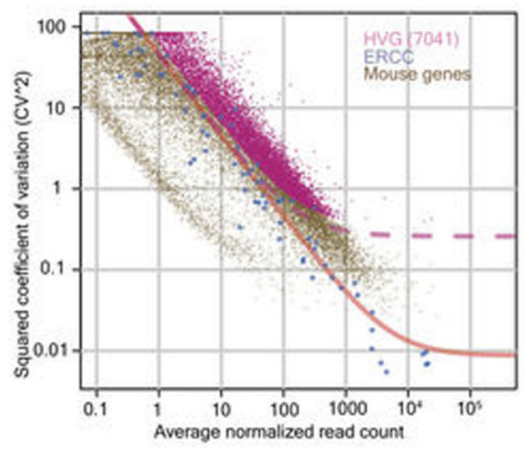

b

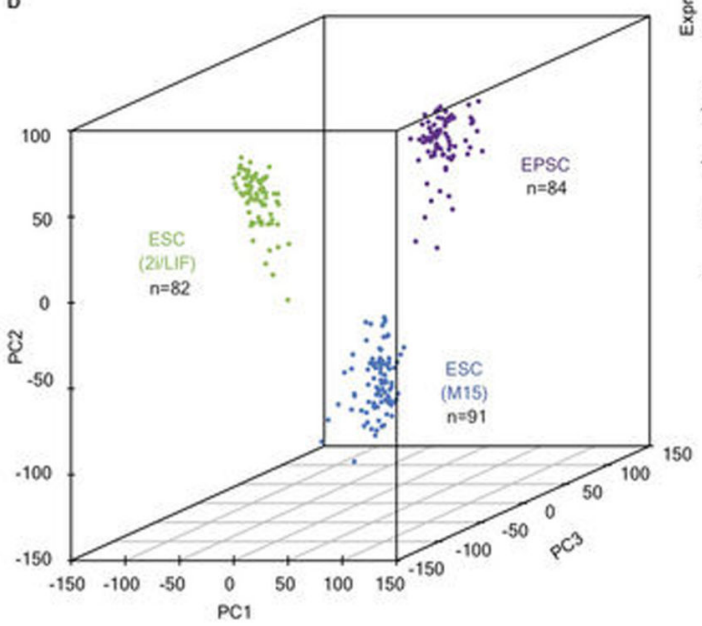

C

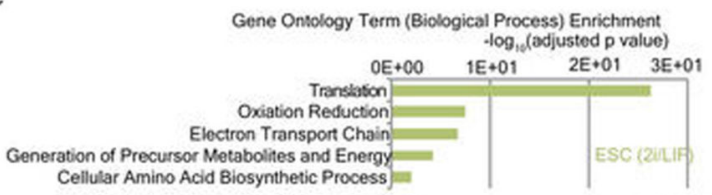

$O E+00 \quad 2 E+00 \quad 4 E+00 \quad 6 E+00 \quad 8 E+00$

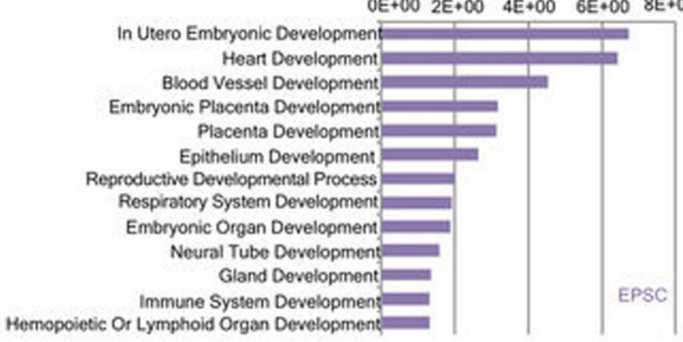

d
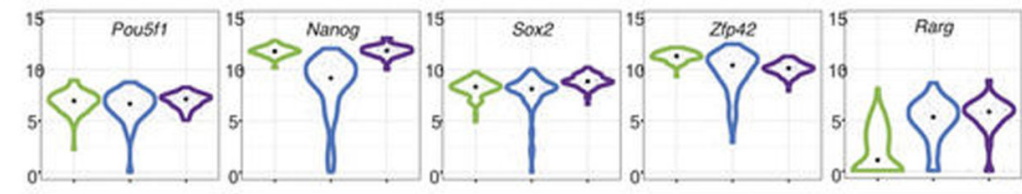

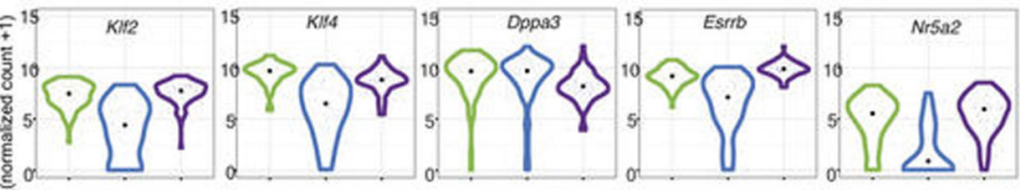
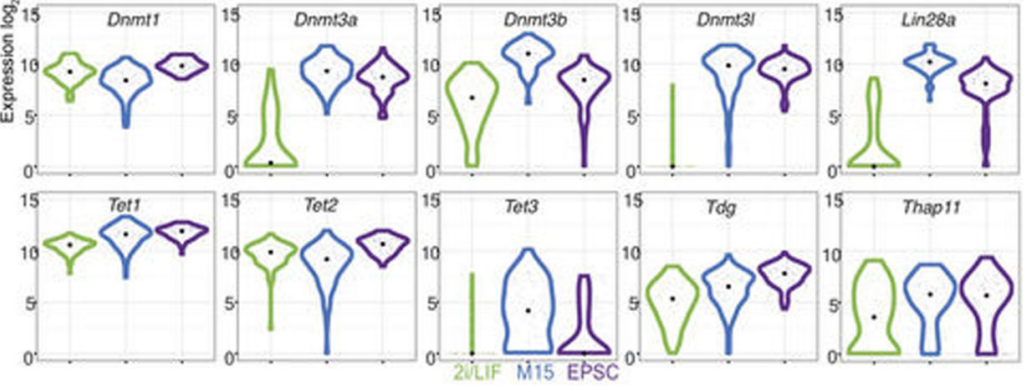

e

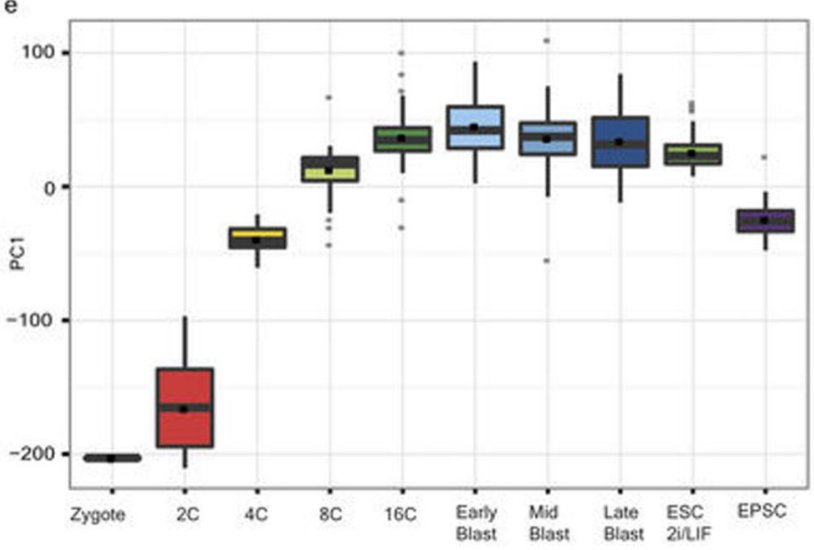

Ext data Fig 7.

a, Scatter plot showing the relationship of EPSC gene expression variability with expression levels in scRNA-seq data. The magenta dots represent genes that showed significantly higher variability (adjusted $P<0.1$ ) than would be expected from the external RNA spike-ins (blue dots). b. Three-dimensional scatter plot showing the separation of 2i/LIF ES cells, M15 ES cells and EPSCs in PCA of scRNA-seq data. Each dot represents a cell. c, Bar graph showing Gene Ontology terms enriched in differentially expressed genes between 2i/LIF ES cells and EPSCs in scRNA-seq data. d, Violin plots comparing expression of selected 
pluripotency regulators, DNA methylation regulators and two-cell embryo-associated genes in 2i/LIF ES cells, M15 ES cells and EPSCs in scRNA-seq data. The dot marks the median of the data. EPSCs $(n=84)$; 2i/LIF ES cells $(n=82)$; M15 ES cells $(n=91)$. e, Box plot comparing the distribution of pre-implantation embryos, 2i/LIF ES cells and EPSCs in the first principal component in PCA. The bar and the small square in the boxes mark the median and mean scores of each group. The upper and lower hinge, and the upper and lower whiskers, mark the first and third quartiles of the data, and the smallest and largest observation excluding outliers, respectively. Outliers were determined as observations less than the first quartile $-1.5 \times$ interquartile range and observations larger than the third quartile $+1.5 \times$ interquartile range. The PCA was performed with differentially expressed genes between EPSCs and 2i/LIF ES cells. 

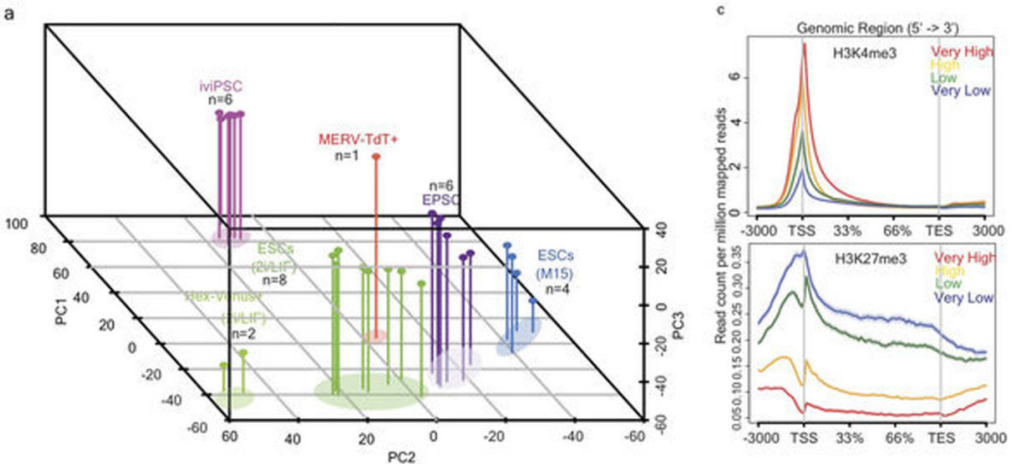

b

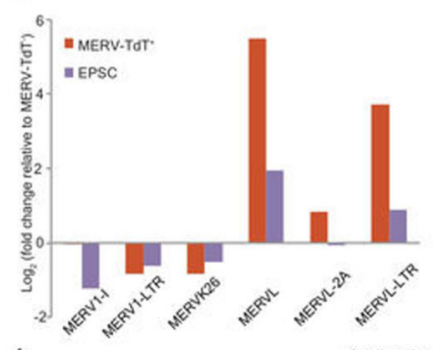

e

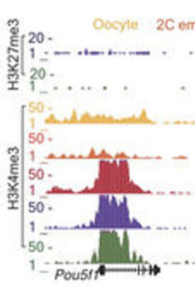

है 20. Oocyie 2 Cembryos 8 Cembryos EPSCS ESC:

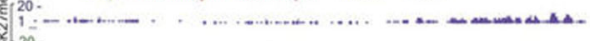

- ........ - - - - -

$-$
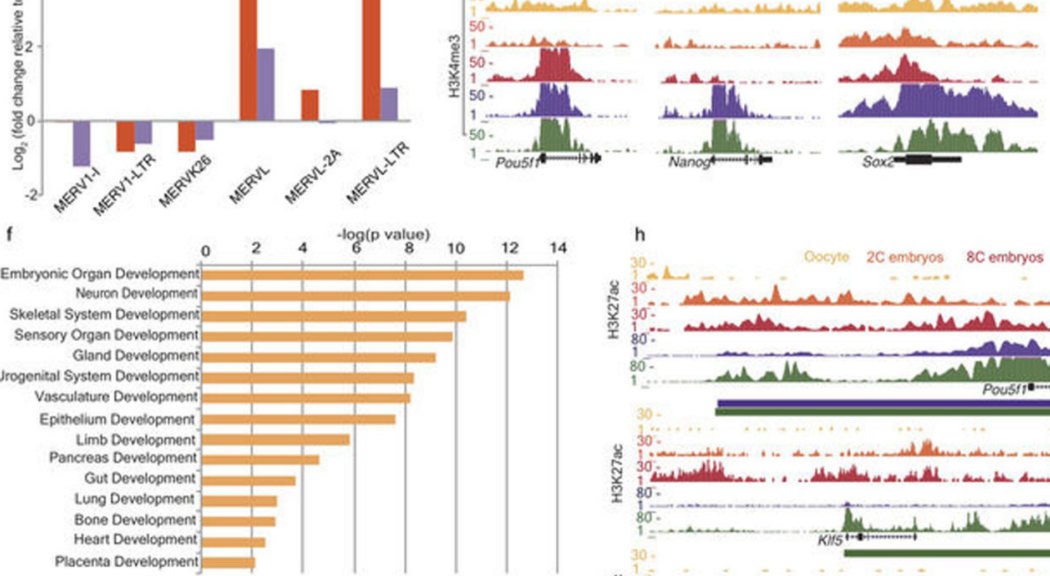

Placentsa Develosonin

Pacenta Develooment

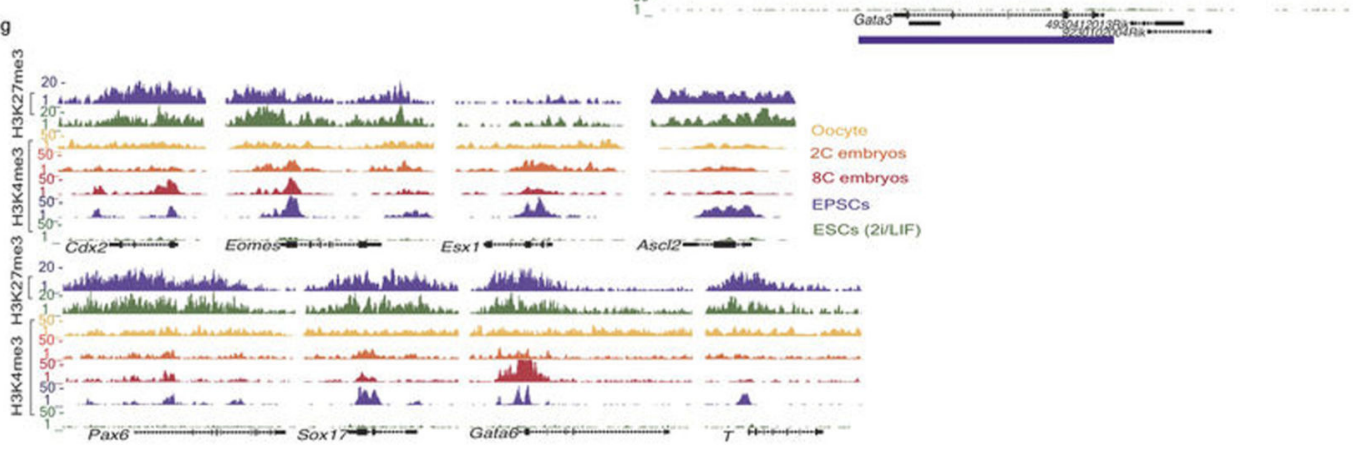

Ext data Fig 8.

a, Three-dimensional scatter plot showing the separation of Hex-Venus ${ }^{+} 2 \mathrm{i} / \mathrm{LIF}$ ES cells, MERV-TdT ${ }^{+}$ES cells, in vivo iPS cells (iviPS cell), 2i/LIF ES cells, M15 ES cells and EPSCs in PCA of bulk RNA-seq data. b, Bar graph comparing the relative expression levels of MERV transcripts in EPSCs and MERV-TdT ${ }^{+}$ES cells to MERV-TdT ${ }^{-}$ES cells. c, Signal intensity distribution of EPSC H3K4me3 and H3K27me3 modification over gene bodies and $3 \mathrm{~kb}$ up- and downstream of the transcription start site (TSS) and transcription end site (TSE). Genes are classified into 'very high', 'high', 'low' and 'very low', depending on their 
length-corrected mean-normalized count quartiles in the single-cell RNA-seq dataset. d, Distribution of H3K27me3 (left) and H3K4me3 (right) signals at gene promoters $( \pm 3 \mathrm{~kb}$ from TSS). The promoters were ranked on the basis of their length-corrected meannormalized count levels in the single-cell RNA-seq dataset. The signals were quantified as read count per million mapped reads. e, Profiles of H3K4me3 and H3K27me3 of 2i/LIF ES cells and EPSCs at selected pluripotency-associated loci. f, Bar graph showing $-\log (P$ value $)$ of each development-related Gene Ontology term enriched in EPSC-specific bivalent genes only. g, Profiles of H3K4me3 and H3K27me3 at selected loci in pre-implantation embryos, EPSCs and 2i/LIF ES cells. Note the similar H3K27me3 profiles in EPSCs and in two-cell or eight-cell embryos. h, Profiles of H3K27ac signals of oocyte, two-cell embryos, eight-cell embryos, 2i/LIF ES cells and EPSCs in selected super-enhancer-associated genes, including the Oct4, KIf5 and Gata3 loci. Note that the super-enhancer at the Gata3 locus is present in EPSCs and in eight-cell embryos but not in 2i/LIF ES cells. 


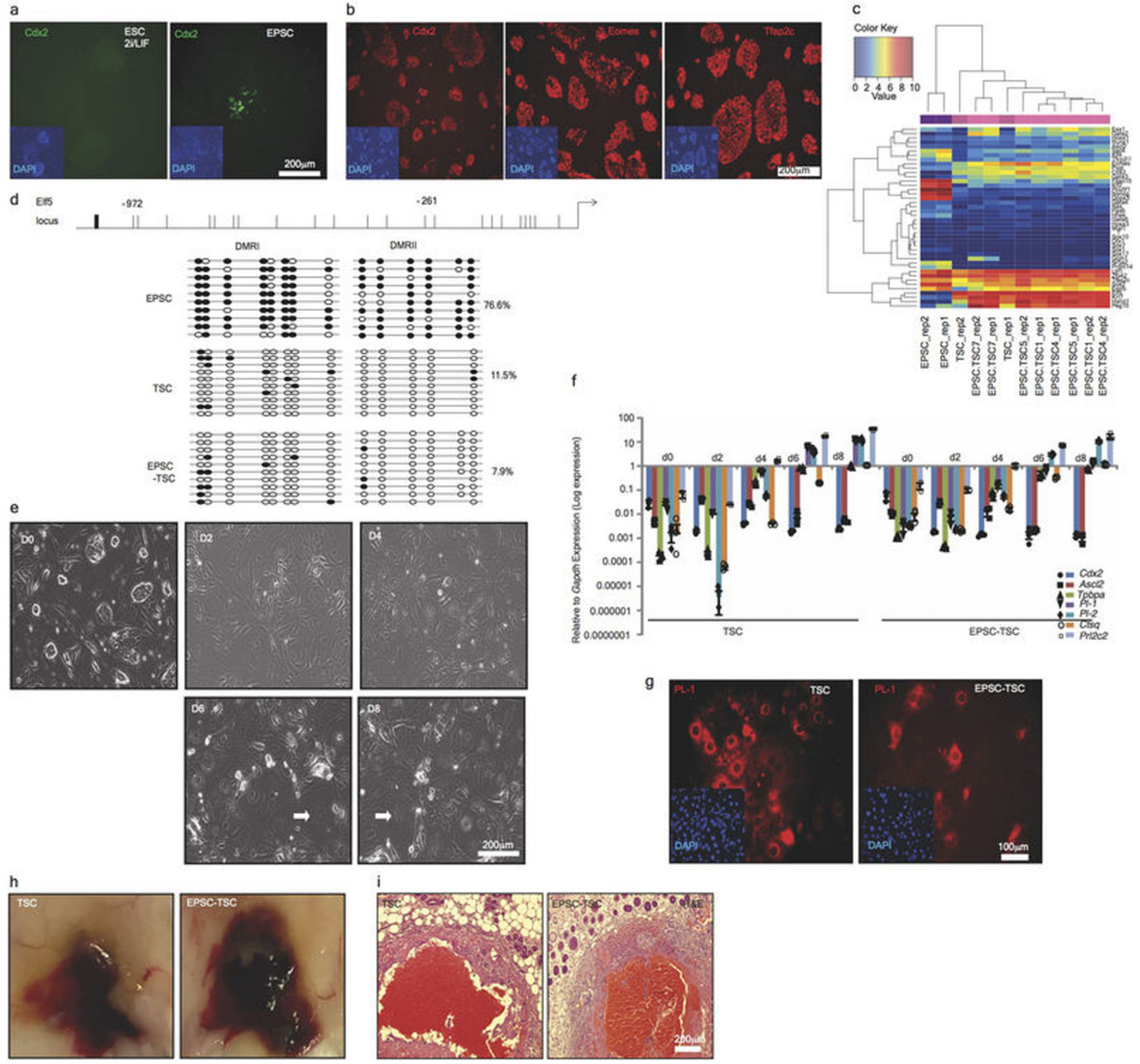

Ext data Fig 9.

a, Immunofluorescence for GFP in EPSCs or 2i/LIF ES cells cultured in TX medium for 6 days. Small patches of $\mathrm{Cdx}^{+}$cells were detected only in differentiated EPSCs, but not 2i/LIF ES cells. DAPI stains the nucleus. The $\mathrm{Cdx} 2^{+}$cells account for around $1.0 \%$ of total cells in flow cytometry. b, Expression of Cdx2, Eomes and Tfap2c in established EPSC TSCs detected by immunostaining. Four EPSC TSC lines were established from Cdx2-GFP EPSCs. c, Heat map comparing the expression of transcription factors of pluripotency, embryo lineages or trophectoderm development in EPSCs, embryo-derived TSCs and multiple lines of EPSC-derived TSCs. The expression levels were calculated in logtransformed values of transcript per million (TPM). EPSC rep1 and rep2 are two biological 
replicates of EPSC RNA samples. d, DNA demethylation at the Elf5 locus in EPSC TSCs ( $n$ =10). $\mathbf{e}$, Phase images of EPSC TSCs in differentiation medium (RPMI 1640 plus $20 \%$ serum without FGF4 and heparin) for the indicated days. Arrows indicate polyploid trophoblasts. f, RT-qPCR analysis of EPSC TSCs or the control TSCs at different days of differentiation. $\mathrm{Cdx} 2$ was downregulated while expression of mature trophoblast genes was upregulated. Expression levels are relative to Gapdh. Data are mean \pm s.d. ( $n=3$ technically independent samples). Overlaid dot plots indicate the distribution of the data. $\mathbf{g}$, Immunofluorescence staining of differentiated EPSC TSCs or TSCs for placenta lactogen-1 (PL-1) (day 8). The insets show DAPI staining results. h, Representative images of haemorrhagic lesions in NSG mice 7 days after subcutaneous injection of EPSC TSCs or TSCs. i, Haematoxylin and eosin sections of haemorrhagic lesions of EPSC TSCs or TSCs. The images show that the well-defined haemorrhagic lesions are lined by large and occasionally multinucleated pleomorphic cells with abundant cytoplasm. These large cells that demarcate the blood-filled space are reminiscent of trophoblast giant cells. 
a
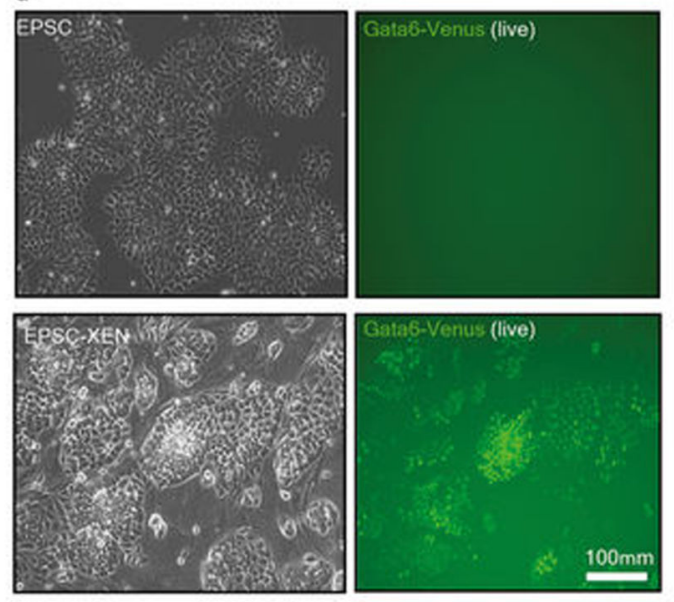

b
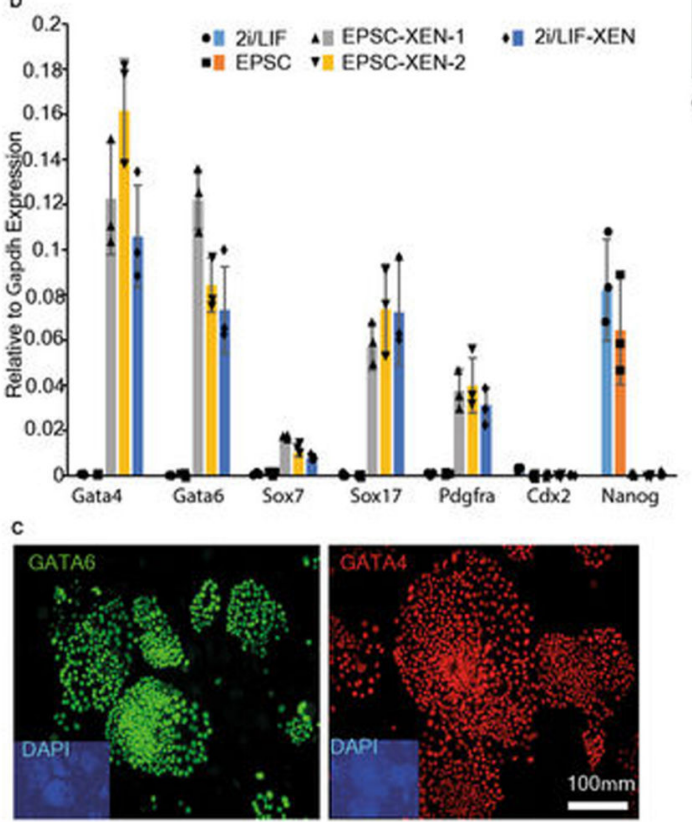

d PrE contribution $(6.5 \mathrm{dpc})$ EPSC-XEN $8 / 19$
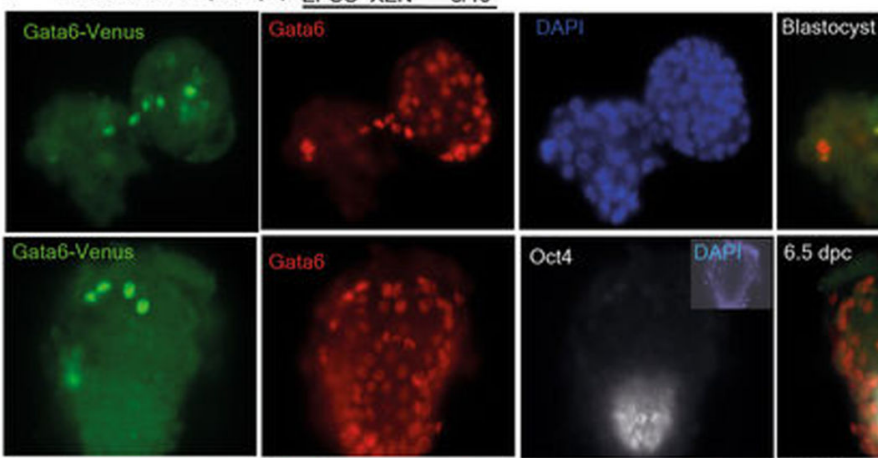

$6.5 d p c$
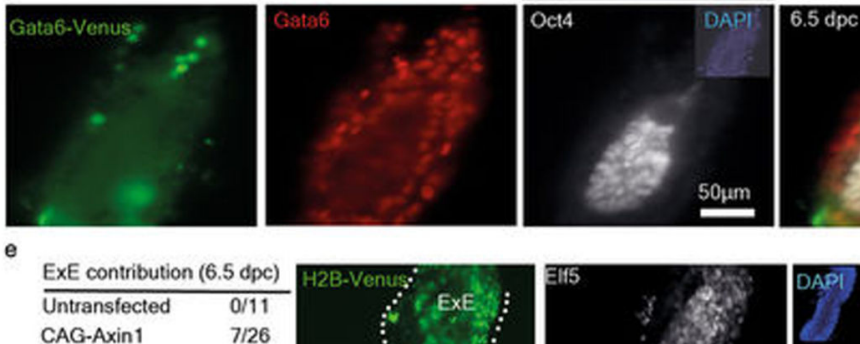
TET-Axin1
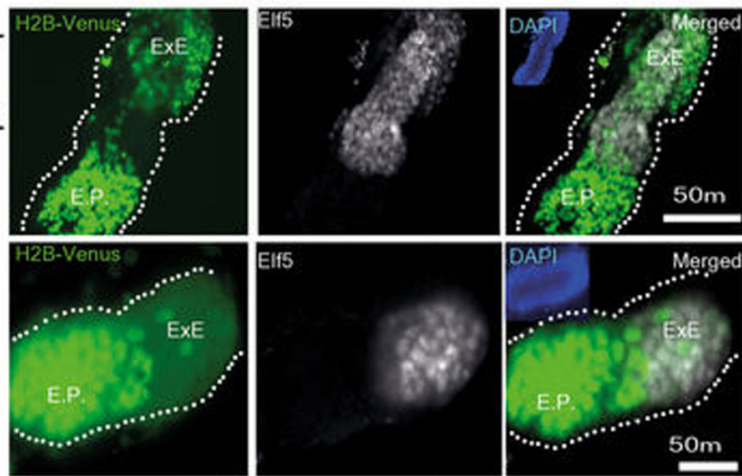

Elf5
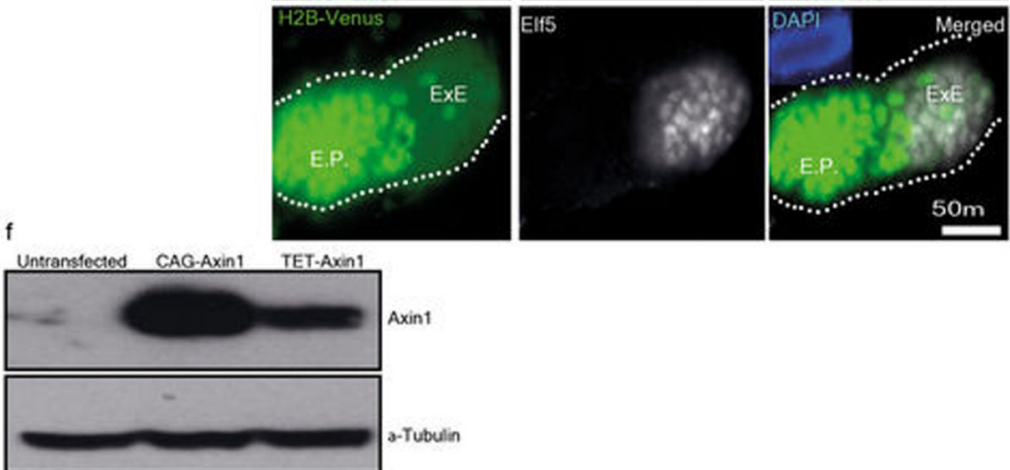

Ext data fig 10.

a, Live images of Gata6H2B-Venus ${ }^{+}$XEN cells derived directly from EPSCs in vitro. $\mathbf{b}$, RT-qPCR analysis of expression of cell-type-specific genes for XEN (Gata4, Gata6, Sox 7 and Sox 17), TSC ( $C d x 2)$ or ES cells (Nanog). Expression levels are relative to Gapdh. Data are mean \pm s.d. ( $n=3$ technically independent samples). Two biologically independent EPSC-XEN cell lines were analysed. Overlaid dot plots indicate the distribution of the data. c, Expression of Gata4 and Gata6 in XEN cells visualized by immunostaining. d, Contribution of Gata6H2B-Venus EPSC-XEN cells in blastocysts $(n=17)$ and in 6.5 d.p.c. chimaeras. The donor cells in the PrE of the hatching blastocyst or in the extra-embryonic endoderm were immunostained by the antibody to Gata6. The Oct 4 antibody staining marks the embryo proper. Inset table shows the contribution. e, Contribution of ES cells overexpressing axin1 in the ExE region in 6.5 d.p.c. chimaeras. Top panels, donor ES cells in the chimaera expressed axin1 under control of the Tet/On system before injection, whereas in the bottom panels the donor cells expressed axin 1 from the CAG promoter. Inset 
table shows the contribution. f, Western blot confirming axin 1 overexpression in ES cells using either the CAG promoter or the Tet/On system. The gel source data are in Supplementary Fig. 1.

\section{Supplementary Material}

Refer to Web version on PubMed Central for supplementary material.

\section{Acknowledgements}

We thank colleagues of the Research Support Facility (B. Doe, S. Newman, E. Grau and others), Y. Hooks, Sequencing (N. Smerdon) and FACS core facilities (B. L. Ng and J. Graham) at the Sanger Institute, the animal facility at the Cancer Research UK Cambridge Institute, and P. Humphreys of the University of Cambridge, for technical support; S. Gerety for the fluorescence stereo microscope, J. K. Kim for informatics advice, S. Rice for help on DNA bisulfite sequencing analysis; J. Nichols, A. Martinez Arias, K. McDole and Y. Zheng for reagents; J. Thomson, E. Robertson and A. Ang for comments. We acknowledge the following funding and support: Wellcome Trust Clinical PhD Fellowship for Academic Clinicians (D.J.R.); PhD fellowship (Portuguese Foundation for Science and Technology, FCT (SFRH/BD/84964/2012)) (L.A.); Japan Society for the Promotion of Science fellowship (Y.T.); National Institutes of Health (RP-PG-0310-10002) (A.C.W.); European Molecular Biology Organization (ALTF938-2014) and Marie Sklodowska-Curie Individual Fellowship (M.A.E.-M.); Biotechnology and Biological Sciences Research Council (BB/K010867/1) and Wellcome Trust (095645/Z/11/Z) (W.R.); Bloodwise (12029), Cancer Research UK (C1163/A12765 and C1163/A21762) and Wellcome Trust core funding (SCI 097922/Z/11/Z) (B.G.); Leading Advanced Projects for Medical Innovation, Japan Agency for Medical Research and Development (H.N. and H.M.); National Health and Medical Research Council Senior Principal Research Fellowship (1110751) (P.P.L.T.); National Natural Science Foundation of China (81671579, 31370904, 30972691) and The National Key Research and Development Program (2017YFA0104500) (L.L.). P.L. thanks M. Stratton, A. Bradley, N. Copeland, N. Jenkins and J. Lupski for their encouragement in these experiments. P.L. is an affiliate faculty member of the Wellcome Trust-MRC Stem Cell Institute, University of Cambridge. The P.L. laboratory is supported by the Wellcome Trust (grant numbers 098051 and 206194).

\section{References}

1. Evans MJ, Kaufman MH. Establishment in culture of pluripotential cells from mouse embryos. Nature. 1981; 292:154-156. [PubMed: 7242681]

2. Tam PP, Rossant J. Mouse embryonic chimeras: tools for studying mammalian development. Development. 2003; 130:6155-6163. [PubMed: 14623817]

3. Saba-El-Leil MK, et al. An essential function of the mitogen-activated protein kinase Erk2 in mouse trophoblast development. EMBO Rep. 2003; 4:964-968. [PubMed: 14502223]

4. Nishioka N, et al. The Hippo signaling pathway components Lats and Yap pattern Tead4 activity to distinguish mouse trophectoderm from inner cell mass. Dev Cell. 2009; 16:398-410. [PubMed: 19289085]

5. Li X, et al. Calcineurin-NFAT signaling critically regulates early lineage specification in mouse embryonic stem cells and embryos. Cell Stem Cell. 2011; 8:46-58. [PubMed: 21211781]

6. Hemberger M, et al. Parp1-deficiency induces differentiation of ES cells into trophoblast derivatives. Dev Biol. 2003; 257:371-381. [PubMed: 12729565]

7. Koh DW, et al. Failure to degrade poly(ADP-ribose) causes increased sensitivity to cytotoxicity and early embryonic lethality. Proc Natl Acad Sci USA. 2004; 101:17699-17704. [PubMed: 15591342]

8. Loh KM, van Amerongen R, Nusse R. Generating cellular diversity and spatial form: Wnt signaling and the evolution of multicellular animals. Dev Cell. 2016; 38:643-655. [PubMed: 27676437]

9. Kapoor A, et al. Yap1 activation enables bypass of oncogenic Kras addiction in pancreatic cancer. Cell. 2014; 158:185-197. [PubMed: 24954535]

10. Shao DD, et al. KRAS and YAP1 converge to regulate EMT and tumor survival. Cell. 2014; 158:171-184. [PubMed: 24954536]

11. Azzolin L, et al. YAP/TAZ incorporation in the $\beta$-catenin destruction complex orchestrates the Wnt response. Cell. 2014; 158:157-170. [PubMed: 24976009] 
12. Wilson MB, Schreiner SJ, Choi HJ, Kamens J, Smithgall TE. Selective pyrrolo-pyrimidine inhibitors reveal a necessary role for Src family kinases in Bcr-Abl signal transduction and oncogenesis. Oncogene. 2002; 21:8075-8088. [PubMed: 12444544]

13. Huang SM, et al. Tankyrase inhibition stabilizes axin and antagonizes Wnt signalling. Nature. 2009; 461:614-620. [PubMed: 19759537]

14. Wang W, et al. Tankyrase inhibitors target YAP by stabilizing angiomotin family proteins. Cell Reports. 2015; 13:524-532. [PubMed: 26456820]

15. Martello G, et al. Esrrb is a pivotal target of the Gsk3/Tcf3 axis regulating embryonic stem cell self-renewal. Cell Stem Cell. 2012; 11:491-504. [PubMed: 23040478]

16. Ying QL, et al. The ground state of embryonic stem cell self-renewal. Nature. 2008; 453:519-523. [PubMed: 18497825]

17. Kim $\mathrm{H}$, et al. Modulation of $\beta$-catenin function maintains mouse epiblast stem cell and human embryonic stem cell self-renewal. Nat Commun. 2013; 4:2403. [PubMed: 23985566]

18. Zimmerlin L, et al. Tankyrase inhibition promotes a stable human naïve pluripotent state with improved functionality. Development. 2016; 143:4368-4380. [PubMed: 27660325]

19. Morgani SM, et al. Totipotent embryonic stem cells arise in ground-state culture conditions. Cell Reports. 2013; 3:1945-1957. [PubMed: 23746443]

20. Latos PA, Hemberger M. From the stem of the placental tree: trophoblast stem cells and their progeny. Development. 2016; 143:3650-3660. [PubMed: 27802134]

21. Kolodziejczyk AA, et al. Single cell RNA-sequencing of pluripotent states unlocks modular transcriptional variation. Cell Stem Cell. 2015; 17:471-485. [PubMed: 26431182]

22. Marks $\mathrm{H}$, et al. The transcriptional and epigenomic foundations of ground state pluripotency. Cell. 2012; 149:590-604. [PubMed: 22541430]

23. Deng Q, Ramsköld D, Reinius B, Sandberg R. Single-cell RNA-seq reveals dynamic, random monoallelic gene expression in mammalian cells. Science. 2014; 343:193-196. [PubMed: 24408435]

24. Wu J, et al. The landscape of accessible chromatin in mammalian preimplantation embryos. Nature. 2016; 534:652-657. [PubMed: 27309802]

25. Macfarlan TS, et al. Embryonic stem cell potency fluctuates with endogenous retrovirus activity. Nature. 2012; 487:57-63. [PubMed: 22722858]

26. Abad M, et al. Reprogramming in vivo produces teratomas and iPS cells with totipotency features. Nature. 2013; 502:340-345. [PubMed: 24025773]

27. Leitch HG, et al. Naive pluripotency is associated with global DNA hypomethylation. Nat Struct Mol Biol. 2013; 20:311-316. [PubMed: 23416945]

28. Dahl JA, et al. Broad histone H3K4me3 domains in mouse oocytes modulate maternal-to-zygotic transition. Nature. 2016; 537:548-552. [PubMed: 27626377]

29. Kubaczka C, et al. Derivation and maintenance of murine trophoblast stem cells under defined conditions. Stem Cell Reports. 2014; 2:232-242. [PubMed: 24527396]

30. Niakan KK, Schrode N, Cho LT, Hadjantonakis AK. Derivation of extraembryonic endoderm stem (XEN) cells from mouse embryos and embryonic stem cells. Nat Protoc. 2013; 8:1028-1041. [PubMed: 23640167] 
a

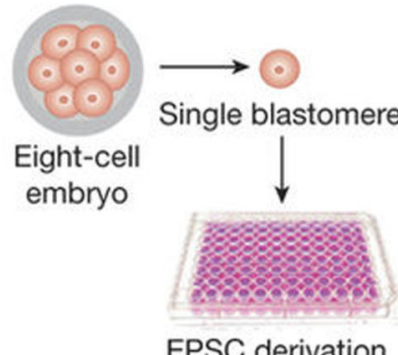

EPSC derivation
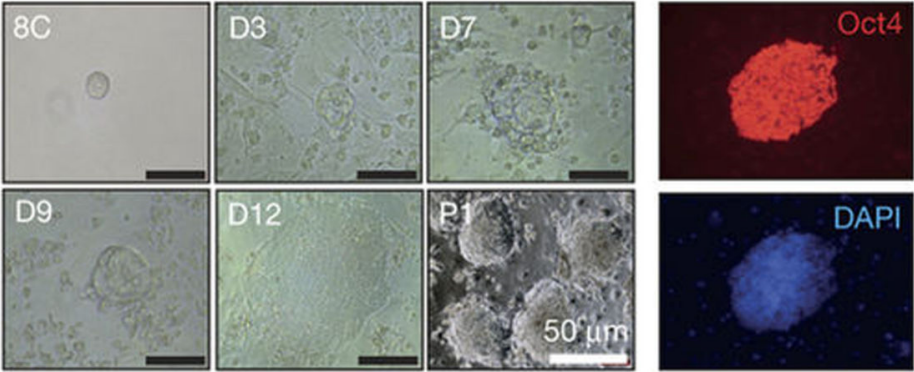

b DR10-EPSC

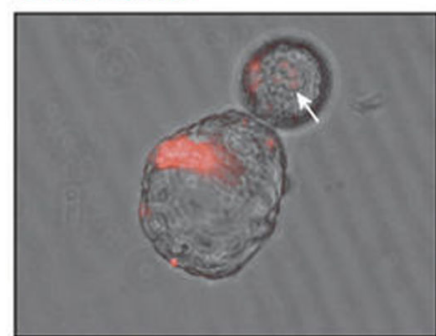

c
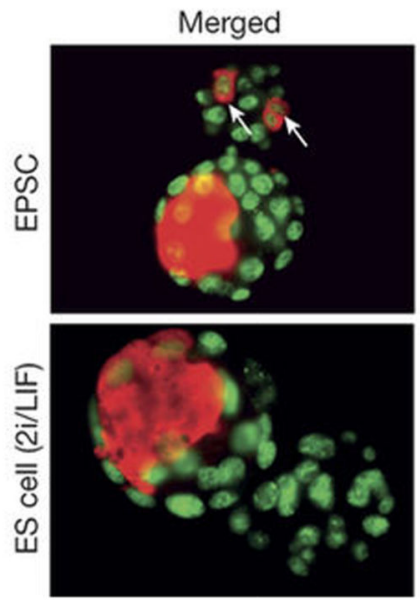

iPS cell-EPSC
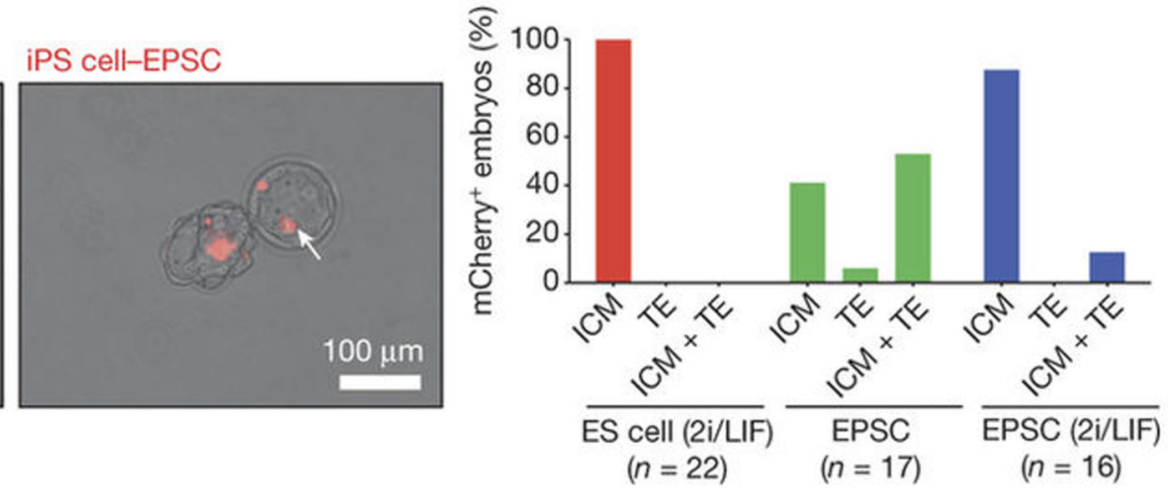
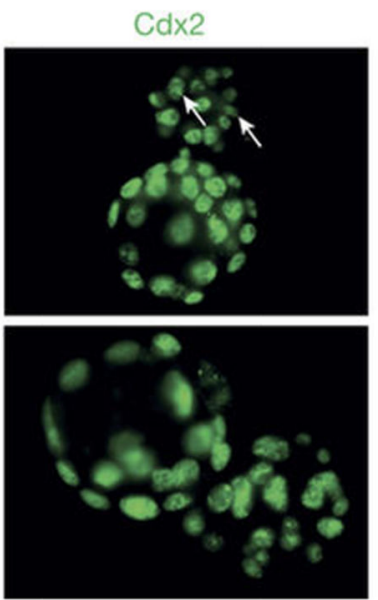

mCherry
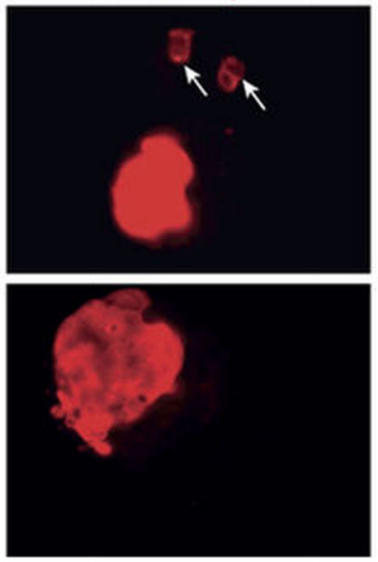
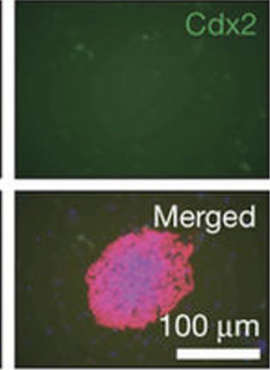

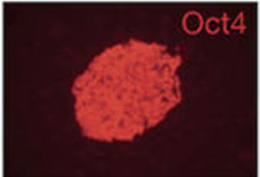

Figure 1.

a, Derivation of EPSC lines from single blastomeres. 8C, eight-cell blastomere; D, day; P1, passage 1. Right: immunostaining for Oct4 and Cdx2 of a primary outgrowth. b, Merged live images of trophectoderm contribution of EPSCs $\left(\mathrm{mCherry}^{+}\right)$in the blastocyst and the contribution percentages. Arrows point to $\mathrm{mCherry}^{+}$cells in the trophectoderm. c, The blastocysts (hatching) developing from morula injected with ES cells or EPSCs were stained for mCherry (in the cytoplasm) and Cdx2 (in the nucleus). DAPI stains the nucleus. The arrows indicate $\mathrm{Cdx} 2^{+} \mathrm{mCherry}^{+}$donor cells. $n=23$ for ES cell (2i/LIF); 35 for EPSC. 


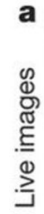
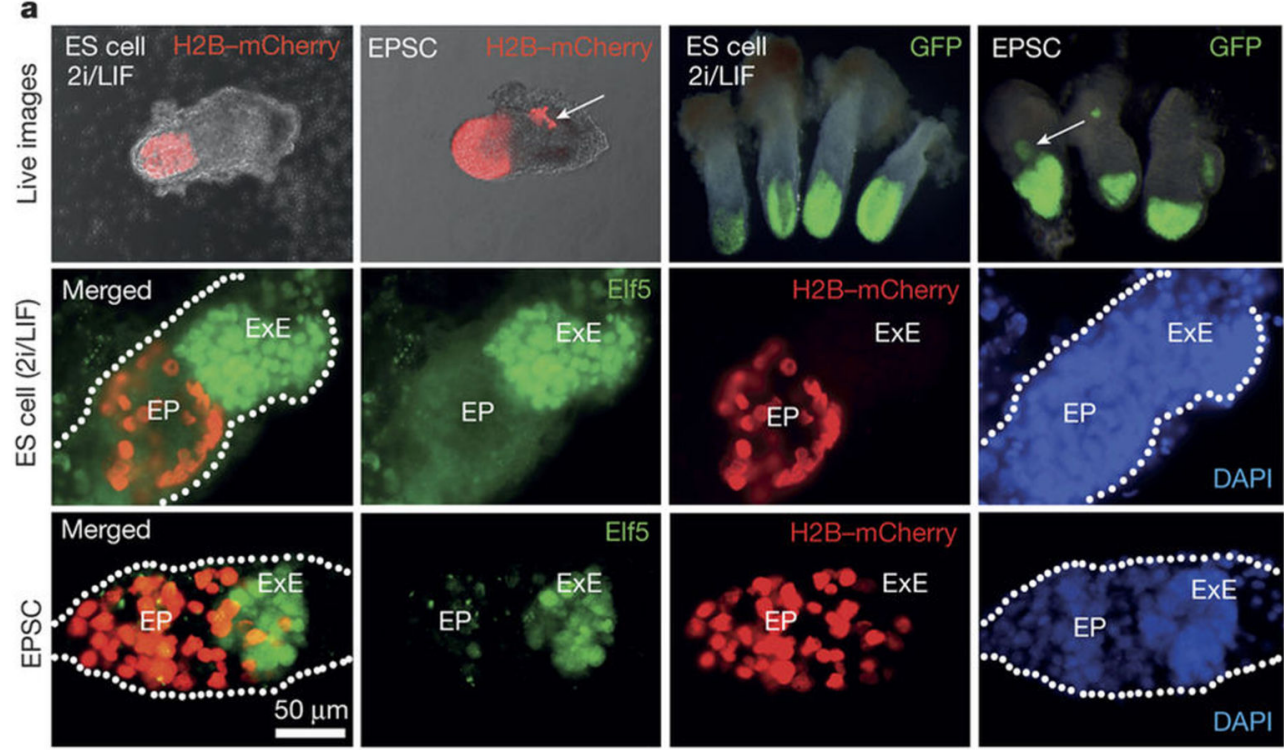

b

C
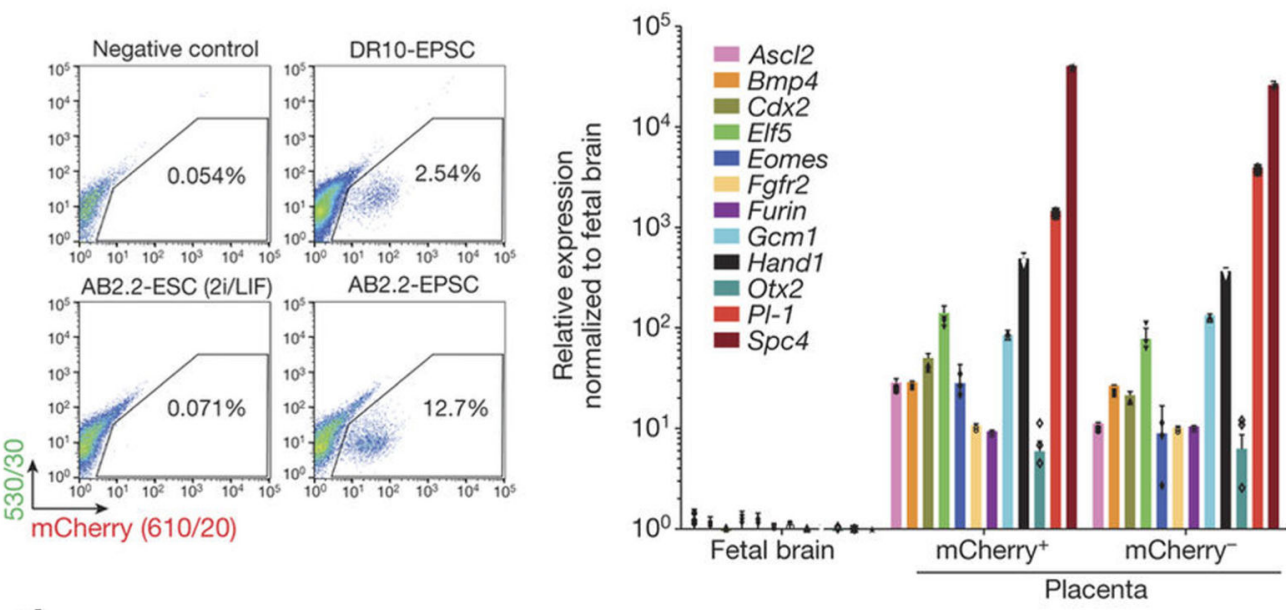

d
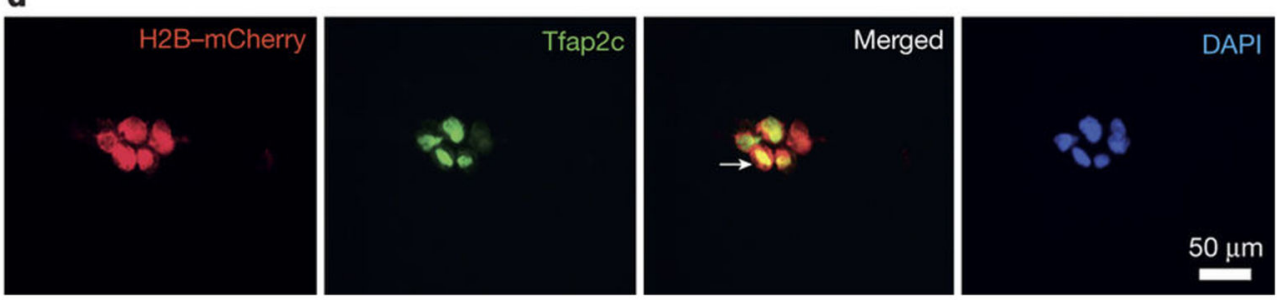

Figure 2.

a, Whole-mount fluorescence imaging of representative 6.0-6.5 d.p.c. ES cell or EPSC chimaeric embryos produced in Cambridge, UK (AB2.2-H2B-mCherry) or at University of Tokyo (BDF1-eGFP). The top panels are four live images. The rest of the chimaeras show immunofluorescence staining for mCherry and Elf5. DAPI stains the nucleus. Chimaeras are marked by dashed lines. Arrows point to mCherry ${ }^{+}$cells in ExE. EP, embryo proper. b, Detection of EPSC-derived mCherry $^{+}$placenta cells in 14.5 d.p.c. chimaeras in flow cytometry. c, Expression of trophoblast genes in $\mathrm{mCherry}^{+}$and $\mathrm{mCherry}^{-}$placenta cells 
from an EPSC 14.5 d.p.c. chimaera. Expression is relative to $A c t b$ and normalized to fetal brain expression. Data are mean \pm s.d. $(n=3)$. Overlaid dot plots indicate the distribution of the data. Pl-1 is also known as Prl3d1; Spc4 is also known as Pcsk6. d, Detection of Tfap2c in $\mathrm{mCherry}^{+}$cells sorted from 14.5 d.p.c. EPSC chimaera placenta. The sorted cells were stained for mCherry and Tfap2c. The experiments were repeated at least three times. Arrow points to the mCherry ${ }^{+} \mathrm{Tfap} 2 \mathrm{c}^{+}$cells. 
a
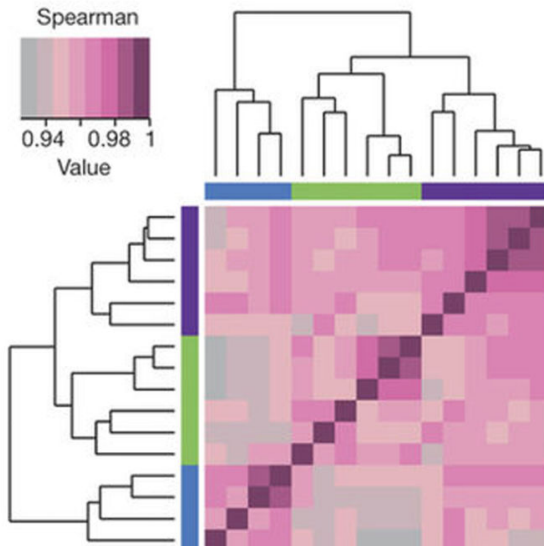

b

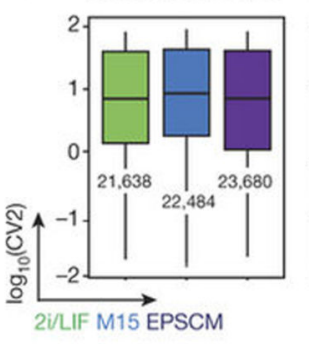

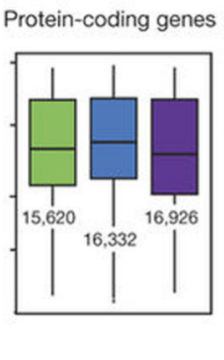

DR9-EPSCM DR25-EPSCM DR10-EPSCM E14-EPSCM AB2.2-EPSCM DR25-2I/LIF DR4-2i/LIF DR9-2i/LIF E14-2i/LIF DR10-2:/LIF DR25-M15 DR10-M15 E14-M15 AB2.2-M15

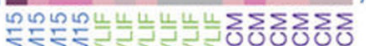

$\sum \sum \sum \sum 5 S S S 300000$

n่tón nand

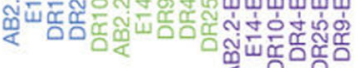

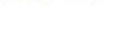

(1)$$
\text { - }
$$

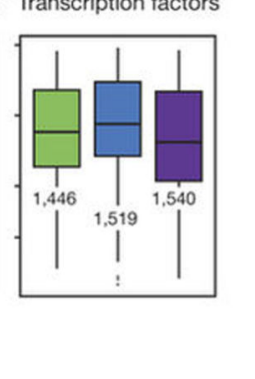

d Normalized gene set enrichment score
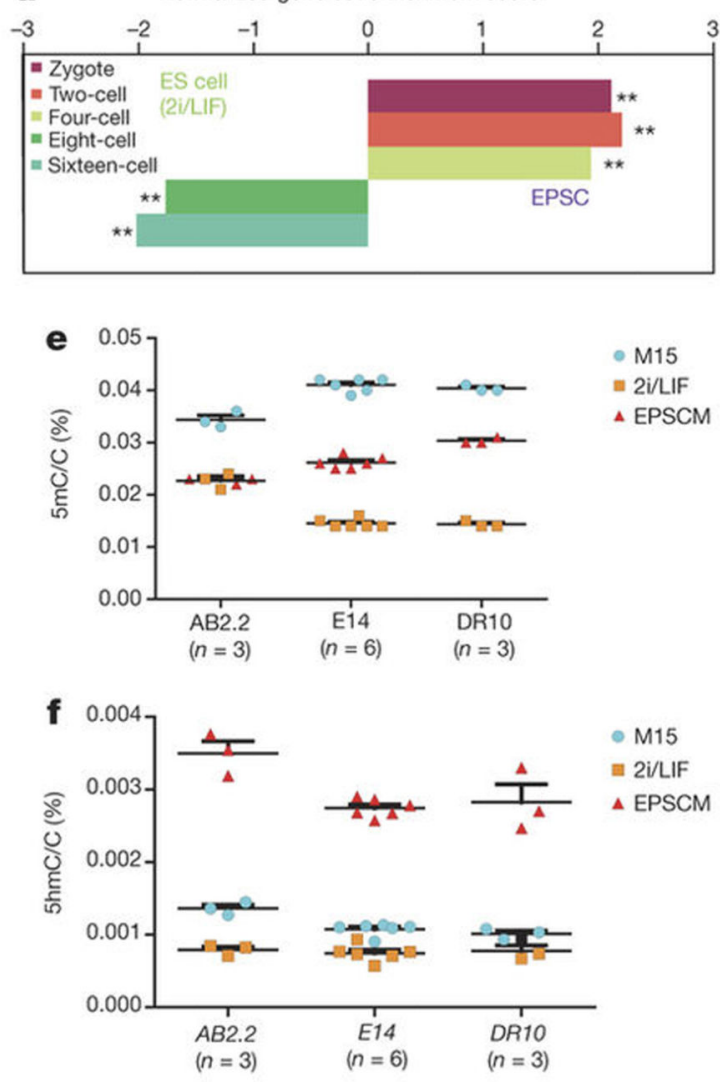
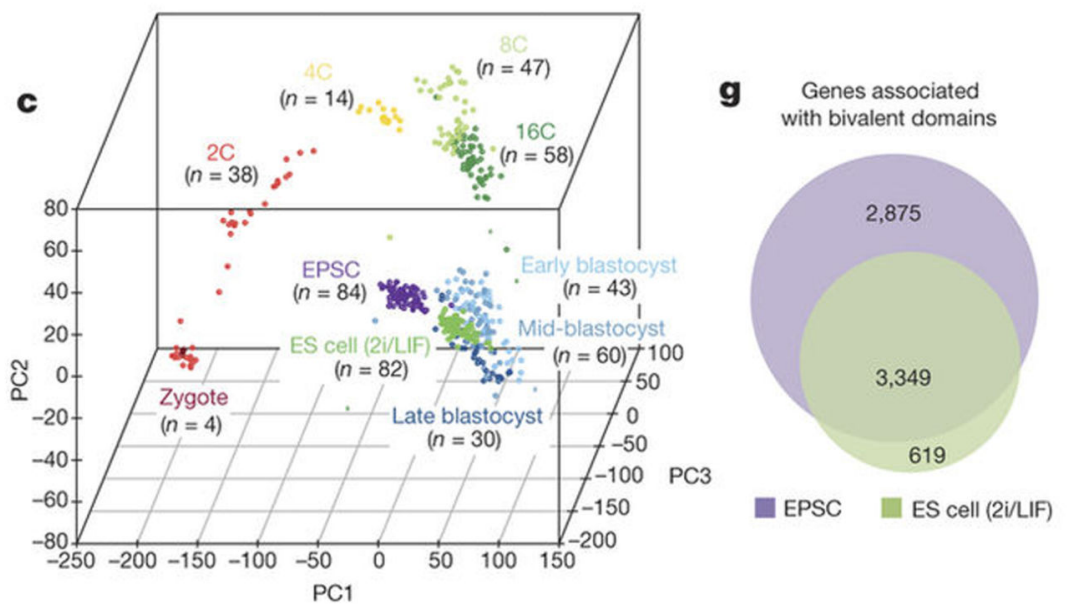

$\mathbf{h}$

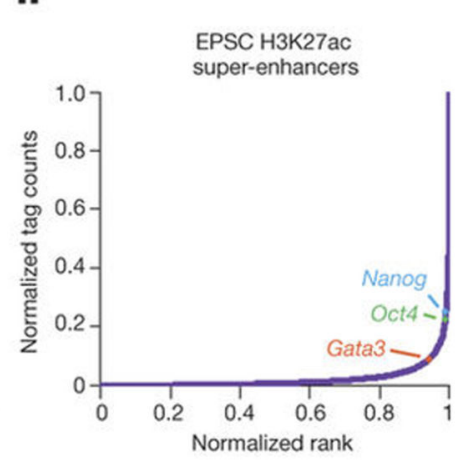

Figure 3.

a, Unsupervised hierarchical clustering of RNA-seq data of mouse ES cell lines (AB2.2 and E14Tg2a), new ES cell lines derived in 2i/LIF media (DR4 and DR9) or EPSC lines derived in EPSCM (DR25 and DR10) from pre-implantation embryos, which were cultured in different media. Inter-sample Spearman correlation coefficient value was shown in the top left corner. b, Box plots comparing the expression variability of the global transcriptome, protein-coding genes and putative transcription factors in EPSCs and ES cells. The bar in the boxes marks the median of each group. The numbers of genes are listed in the panel. c, 
Three-dimensional scatter plot based on PCA of scRNA-seq data, showing the separation of pre-implantation embryos, 2i/LIF ES cells and EPSCs. d, Normalized enrichment scores of different embryonic stage-specific gene sets between EPSCs and 2i/LIF ES cells. **Nominal $P<0.01$. e, f, Grouped scatter charts comparing the fractional levels of methylated $(5 \mathrm{mC})$ (e) and hydroxymethylated $(5 \mathrm{hmC})(\mathbf{f})$ cytosine in EPSCs and in ES cells quantified by mass spectrometry. C, cytosine. g, Venn diagram comparing the number of bivalent histone domain-associated genes. h, Super-enhancers in EPSCs. H3K27ac peak regions associated with Gata3, Oct4 or Nanog are marked. Details of informatics and statistical analyses are described in Methods. 

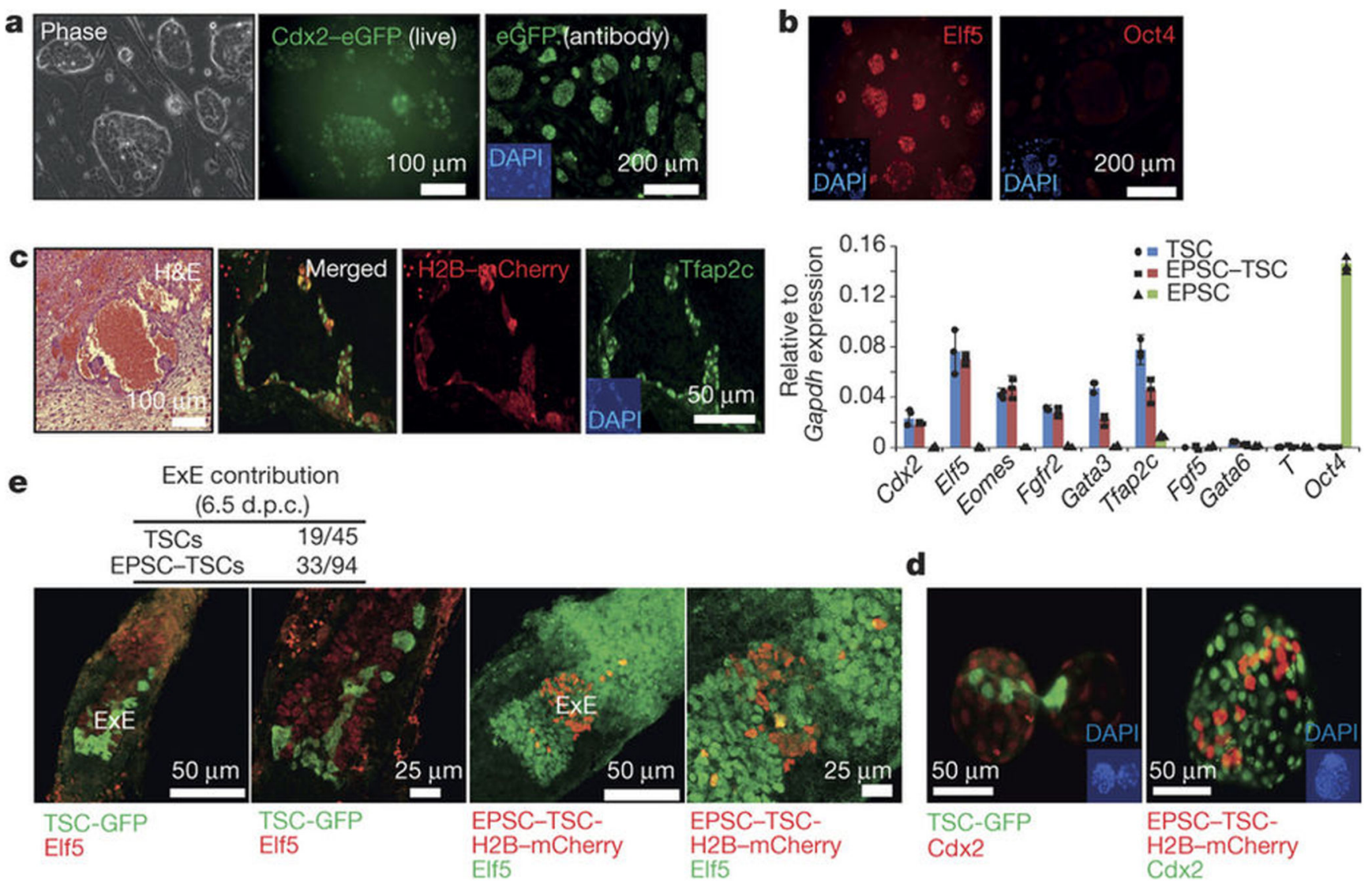

d

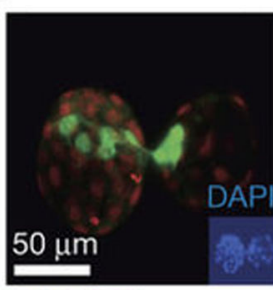

TSC-GFP

Cdx2

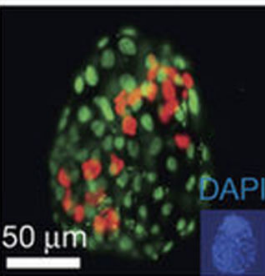

EPSC-TSC-

H2B-mCherry

$\mathrm{Cdx} 2$

Figure 4.

a, Morphology and GFP expression in established Cdx2-GFP EPSC TSCs in live and immunostained images. DAPI stains the nucleus. b, Immunostaining and RT-qPCR detection of key TSC genes and of Oct4 in EPSC TSCs. Expression levels are relative to Gapdh. Data are mean \pm s.d. $(n=3)$. Overlaid dot plots indicate the distribution of the data. c, Histology and immunostaining of sections of haemorrhagic lesions from EPSC TSCs. DAPI stains the nucleus. H\&E, haematoxylin and eosin. d, Localization of TSC-derived cells in the blastocysts from injected morulas. Some donor cells expressed Cdx2. $n=28$ blastocysts for TSCs; 38 for EPSC TSCs. e, Confocal microscopy images of TSCs (cytoplasmic GFP) or EPSC TSCs (H2B-mCherry) in the Elf5-expressing ExE in the 6.5 d.p.c. chimaeras. Experiments were repeated at least three times. The table lists the chimaeras versus total dissected embryos. 